\title{
Modelo de evaluación de proyectos de inversión en condiciones de riesgo para apertura de programas de pregrado en instituciones de educación superior de Colombia: caso de estudio
}

\author{
Sandra Milena Toro*, Jhon Edwin Ledezma**, John Willmer Escobar*** \\ Pontificia Universidad Javeriana Cali. Colombia. \\ Recibido: 6 de abril de 2015 / Aprobado: 10 de junio de 2015
}

Resumen: Se propone un modelo de evaluación de proyectos de inversión bajo condiciones de riesgo en instituciones de educación superior. Es común que estas instituciones se enfrenten al proceso de toma de decisiones en un proyecto de inversión, esperando que el panorama sea lo más acertado posible. Por esto deben contar con herramientas que les permitan una valoración de riesgos alrededor de la inversión. El modelo propuesto ha sido validado con información real y simulado con pruebas computacionales en Crystal Ball®. El desarrollo del modelo ha hecho posible la incorporación y el uso de conceptos de riesgo para el proceso de toma de decisiones.

Palabras clave: riesgo / evaluación de proyectos de inversión / instituciones de educación superior / simulación Montecarlo / Crystal Ball / Colombia

\section{Model Evaluation of Investment Projects Unsafe to open Undergraduate Programs in Higher Education Institutions of Colombia: Case Study}

ABsTRACT: A model for evaluating investment projects under risk conditions in institutions of higher learning is proposed. It is common that these institutions are facing the process of decision making in an investment project, hoping that the picture is as accurate as possible. For this they must have tools that allow them a risk assessment about the investment. The proposed model has been validated with real data and simulated with computational tests Crystal Ball®. The development of the model allowed the incorporation and use of concepts of risk for the process of decision making.

Key words: risk, evaluation of investment projects / institutions of higher education / Monte Carlo simulation / Crystal Ball ${ }^{\circledR}$ / Colombia

Correos electrónicos: *smtoro@javerianacali.edu.co, **met8686@hotmail.com,

***jwescobar@javerianacali.edu.co 


\section{INTRODUCCIÓN}

Durante las últimas tres décadas Colombia se ha visto en la obligación de desafiar a nuevos sectores económicos, lo que le ha permitido incursionar en un mundo de competitividad y globalización; por ello, es importante resaltar la gestión que realizan tanto los sectores públicos como privados para el fortalecimiento de la educación, que haga posible afrontar retos económicos actuales, generando el desarrollo y crecimiento sostenido con una alta eficiencia.

El gobierno nacional colombiano ha establecido como política que la educación sea una herramienta que permita construir un país con paz y equidad, fijándose como meta que Colombia sea el país más educado de América Latina en el año 2025, y como gran reto superar en indicadores internacionales de educación a Chile, Costa Rica, México y Brasil (Schwab y Sala-i-Martin, 2013). No obstante, tanto en el sector público como en el sector privado se optimizan los recursos financieros con el término más conocido como "recorte" en el presupuesto.

Algo muy similar sucede en el ámbito internacional, como se ve en la literatura relacionada con la educación, el desarrollo y la pobreza, en la cual evalúan uno de los principales artículos que presentó la Unicef, donde conservan el pensamiento de que el sector educativo no requiere recortes en su presupuesto sino más bien el compromiso político y una eficiente gestión administrativa que permita mejorar los niveles de competitividad, a pesar de las limitaciones financieras (Cremin y Nakabugo, 2012). Por esta razón, la evaluación de proyectos de inversión en la apertura de nuevos programas en el sector educativo juega un papel importante en nuestro país, en el cual se amerita que se desarrollen trabajos de investigación. El modelo propuesto cumple con un objeto social y educativo, a la vez que permite dar soporte al proceso de toma de decisiones financieras.

Los modelos de decisiones similares se han considerado en el sector educativo de varios países, como por ejemplo en Turquía, el cual ocupa el puesto 44 en el ámbito mundial, y en el pilar de educación superior y entrenamiento el puesto 65, según se expone en The Global Competitiveness Report (Schwab y Sala-i-Martin, 2013). Tal como lo manifiesta Gölpek (2012) en su investigación realizada en Turquía sobre el precio de la educación superior y la demanda individual, donde la decisión de inversión está sujeta a tres factores: posible rentabilidad en el futuro, costo de inversión y tasa de interés, en el que define que existe una 
relación inversa entre las tasas de interés y la capacidad de inversión, y establece que cuando la tasa de interés baja las inversiones aumentan, generando con esto beneficios para la economía del país y oportunidades para tomar decisiones en el marco de las tasas de retorno y de interés.

En este orden de ideas, si el sector educativo de nuestro país asume el desafío de ser más productivo en la toma de decisiones, podría garantizar el aumento del desarrollo económico sostenible, la eficiencia en la toma de decisiones y la optimización del presupuesto para los diferentes proyectos de inversión, los cuales por su origen están enfocados en disminuir la tasa de desescolarización en Colombia; en la medida en que existe una estrecha relación entre el desarrollo, la educación, la salud y la generación de riqueza sostenida, donde el camino más directo para mejorar los niveles de vida es invertir en el bienestar y la educación de las personas, ya que son estas las que no solo garantizan mejores condiciones de vida para ellos mismos y sus familias, sino que también contribuyen a la riqueza y el progreso de la sociedad (Cremin y Nakabugo, 2012).

Basado en lo anterior, y de acuerdo con las políticas educativas nacionales, nuestro trabajo no solo contempla el desarrollo de un modelo de evaluación de proyectos de inversión bajo condiciones de riesgo que le permita a una institución de educación superior valorar el impacto en la toma de decisiones sobre el desempeño financiero relacionado con la apertura de un programa académico, validando resultados con simulación de Montecarlo, sino que también determina el impacto de las decisiones sobre la generación de valor y los niveles de riesgo financiero.

\section{MARCO GENERAL DEL MODELO DE EVALUACIÓN}

En el modelo propuesto se han identificado una serie de elementos necesarios para el desarrollo del objetivo relacionado con la apertura de nuevos programas académicos de pregrado:

a) Identificación del nivel de riesgo. El nivel de riesgo se define como la posibilidad de que las acciones implementadas puedan generar resultados no esperados. En este sentido, la definición de riesgo surge de nuestra incapacidad de predecir con certeza lo que sucederá en el futuro. Contrario a lo que se cree intuitivamente, el riesgo no es ni bueno ni malo, es algo natural, una consecuencia de la incertidumbre del mundo real en que se vive. La aversión humana al riesgo ha 
hecho que las inversiones con mayores riesgos tengan retornos más altos, para así poder seducir a potenciales inversionistas, ya que de otra manera no se "arriesgarían" a invertir, tal como lo manifiestan Ortiz y Ortiz (2009), por lo tanto, el riesgo es también una oportunidad de altos retornos.

b) Consideración de las posibles restricciones. Para el desarrollo de un modelo de evaluación de proyectos de inversión bajo condiciones de riesgo, que permita a una institución de educación superior valorar el impacto en la toma de decisiones sobre el desempeño financiero relacionado con la apertura de un programa académico de pregrado en la jornada diurna, se deben considerar las siguientes restricciones:

- Información estadística sobre algún número de inscritos o aspirantes a programas académicos similares.

- La existencia de pronóstico de demanda de la cantidad de estudiantes para habilitar los programas existentes en jornadas nocturna y diurna.

- El perfil de los aspirantes de los programas académicos para enfocar las estrategias de mercado.

- Bases de datos o información estadística sobre aspirantes a programas similares.

- Base de datos georreferenciada con ubicación, sector, estrato o barrio, que permita obtener información de un sector poblacional para implementar una estrategia de mercado.

c) Identificación de la viabilidad financiera. Para identificar la viabilidad financiera de un proyecto es necesario construir un flujo de caja que permita identificar y analizar los posibles riesgos en las variables críticas, con el fin de ser efectivos en la toma de decisiones. Cuando se trata de una evaluación financiera de proyectos, los datos son muy importantes y los parámetros deben ser muy precisos, así como la identificación de las limitaciones de los métodos de predicción, las condiciones de riesgo y los factores que puedan afectar el flujo de caja del proyecto de inversión (Wenyi, 2008).

d) Selección de los criterios de decisión o variables de salida. Los métodos de decisiones considerados en el modelo de evaluación propuesto son:

- El valor presente neto promedio (VPN promedio). Vélez (2003) lo define como un indicador adecuado para seleccionar alternativas de inversión entre un grupo de alternativas mutuamente excluyentes. Sin embargo, los supuestos que tienen implícitos o 
explícitos no siempre se cumplen y no se conocen. El VPN se puede definir como el valor que recibe en exceso un inversionista sobre su inversión, después de que se ha descontado el interés de la tasa de descuento.

La regla de decisión para el método del VPN, que es un modelo matemático y normativo, indica qué decisión se debe tomar, teniendo en cuenta lo siguiente:

- si el VPN es mayor a cero se debe aceptar

- si el VPN es igual a cero se debe ser indiferente

- si el VPN es menor que cero se debe rechazar

- La desviación estándar de las rentabilidades esperadas del VPN (o). Es definida por Ortiz y Ortiz (2009) como la raíz cuadrada de la varianza, y al igual que esta es una medida de dispersión respecto a la media. Esta métrica sirve para describir la desviación promedio respecto a la media e identificar la desviación de las rentabilidades esperadas del VPN.

Una forma simple de medir el riesgo de un activo es mediante la volatilidad de sus retornos, ya que cuando un activo tiene alta volatilidad su resultado presenta una mayor incertidumbre. Una posible aproximación a la volatilidad es la desviación estándar del activo. Si se supone que los retornos de los activos provienen de una distribución normal, la cual se encuentra definida por su media y su varianza, entonces se puede encontrar con facilidad la probabilidad de que el retorno se encuentre dentro de un rango específico (Melo y Camargo, 2004).

- El valor en riesgo (VaR). Es un método de cuantificación muy popular en el sector financiero, en razón a su sencillez y facilidad del cálculo, partiendo únicamente de la estimación de la volatilidad. Adicionalmente permite obtener un valor numérico de la pérdida esperada para una inversión (Bravo y Sánchez, 2007).

El VaR es definido como la máxima pérdida esperada en un portafolio de activos financieros, dado un cierto nivel de confianza y de manera análoga para un proyecto podría definirse como un VPN en riesgo, un posible resultado del VPN del proyecto dado un cierto nivel de confianza, es decir, el mínimo VPN esperado de un proyecto con un cierto nivel de confianza, definido por los autores Manotas y Toro (2008).

- El valor presente neto en riesgo (VPN en riesgo). Para entender mejor los conceptos establecidos por Bastidas y Ledezma (2012), que adoptan de Manotas y Toro (2008), véase la figura 1, donde 
se explica la estimación del VPN en riesgo con relación al nivel de confianza, pues se generan dos (2) posibles aproximaciones. La primera es calcular el VPN dado un cierto nivel de confianza (VPNa) y la segunda calcular el nivel de confianza (a) para un VPN igual a cero.

Asumiendo que la función de densidad de probabilidad del proyecto es $\mathrm{f}(\mathrm{VPN})$, el VPN en riesgo del proyecto dado un cierto nivel de confianza a se puede definir como el área bajo la curva entre $-\infty$ y VPNa. Por otra parte, el nivel de confianza para un $\mathrm{VPN}=0$ se puede obtener mediante la integración entre $-\infty \mathrm{y}$ cero (0) sobre la función de densidad de probabilidad.

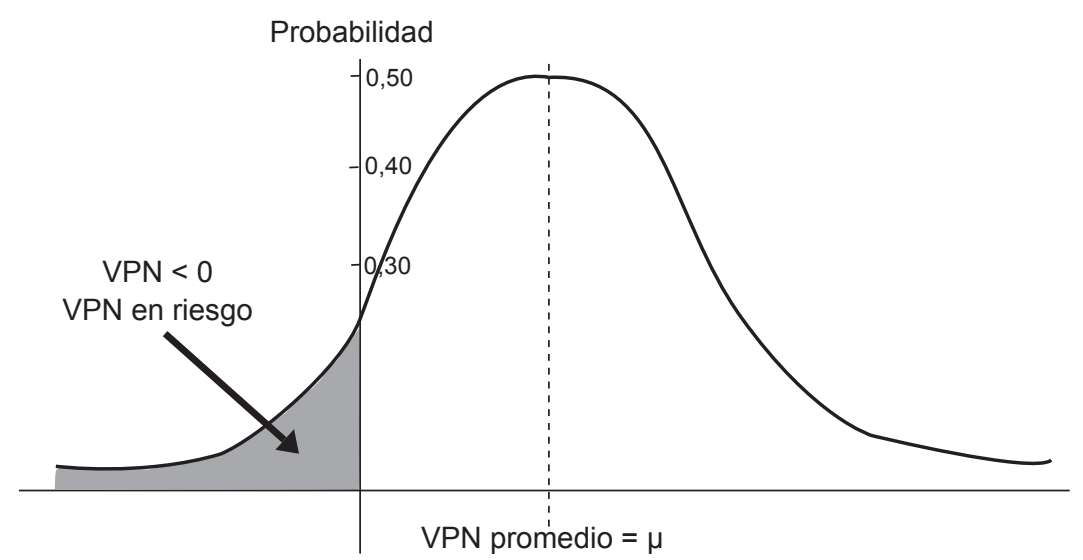

Figura 1. Valor presente neto en riesgo

Fuente: Bastidas y Ledezma (2012)

En ese orden de ideas, el VPN en riesgo se puede definir como:

$$
\mathrm{VPN} \text { en riesgo }=\mathrm{VPN} \text { medio }-\mathrm{Z}(\mathrm{\alpha}) . \sigma
$$

Siendo $\mathrm{Z}(\alpha)$ = número de unidades de la desviación normal estándar que corresponden al nivel de confianza (a), por ejemplo para un nivel de confianza del $95 \%, \mathrm{Z}(\mathrm{\alpha})=1.65$.

- Tasa interna de retorno (TIR). Es una medida porcentual de la magnitud de los beneficios que le genera un proyecto a un inversionista, se toma la decisión teniendo en cuenta la siguiente regla, como lo expone Vélez (2003): 
- si la TIR es mayor que la tasa mínima aceptable, se debe aceptar

- si la TIR es igual a la tasa mínima aceptable, se debe ser indiferente

- si la TIR es menor que la tasa mínima aceptable, se debe rechazar

- Coeficiente de variación o el coeficiente de variabilidad. Se encuentra, según Ortiz y Ortiz (2009), al dividir la desviación estándar entre la media, que es una medida de cuánto varían los datos proyectados respecto de la media. En general, su valor se encuentra entre 0 y 1 y solo en ocasiones, cuando la dispersión es muy grande, supera este rango.

- El valor en riesgo condicional (CVaR). Este indicador mide la pérdida esperada promedio (Jara y Melgar, 2007). Adicionalmente define el CVaR como una medida alternativa al VaR, que cuantifica las pérdidas que se pueden encontrar en las colas de las distribuciones. Como medida de riesgo tiene ventajas significativas frente al $\mathrm{VaR}$ y se deriva también de la distribución de rendimientos de la cartera de activos. Esta métrica es alternativa al VAR y se define como la media de las pérdidas respecto de un nivel de probabilidad $\alpha \%$ en un horizonte de tiempo determinado, es decir, las pérdidas esperadas que pueden darse con esa probabilidad. En consecuencia, el valor del VaR no será nunca mayor del CVaR, por ello, el análisis con bajos valores de CVaR tendrán un VaR aún menor (Bastidas y Ledezma, 2012).

e) Establecimiento del método para evaluar el proyecto de inversión. En el modelo de evaluación propuesto se ha utilizado el análisis de riesgo cuantitativo mediante la simulación Montecarlo, considerando el análisis de sensibilidad y el análisis de escenarios mediante el atributo dinámico de muestreo probabilístico aleatorio con distribución. En la literatura de gestión de proyectos, la simulación Montecarlo se asocia generalmente con la gestión de riesgos, aunque en la práctica también se puede aplicar en la evaluación de la gestión del tiempo (programación) y la gestión de costos (presupuesto). Este método especifica ciertas condiciones y simulaciones de variables del entorno con variables de decisión, ambas claves para los resultados del proyecto, como el VPN o la TIR, con base en los perfiles de riesgo para todas las variables de riesgo relevantes (Bock y Trück, 2011). 


\section{DESCRIPCIÓN DEL MODELO DE EVALUACIÓN DE INVERSIÓN PROPUESTO}

La formulación de un modelo de evaluación de proyectos de inversión bajo condiciones de riesgo para el sector educativo es un área de investigación preponderante. Particularmente, todo el estado del arte con relación a la evaluación de proyectos bajo riesgo se ha desarrollado en torno a la evaluación de proyectos de inversión dedicados al sector productivo o de servicios, pero no directamente vinculados con el sector educativo, con relación al propósito de habilitar un programa académico de pregrado, reduciendo mucho más la información cuando en la investigación se incluyen condiciones como riesgo en la evaluación de proyectos.

Por esta razón, el análisis se ha basado en nuevos y tradicionales métodos para la evaluación de proyectos de inversión, los cuales se resumen como una matriz de enfoques, como se observa en la figura 2 (perspectiva de análisis), donde los análisis son segregados por su perspectiva y por su tipo. Con relación a la perspectiva, el enfoque analítico puede ser de arriba hacia abajo o de abajo hacia arriba. El primero implica un mayor enfoque de las variables macro sobre las variables micro, considerando en este orden de ideas la perspectiva global, la planeación estratégica, las condiciones del mercado o las condiciones del sector educativo, y más concretamente las ventajas competitivas de la institución educativa objeto de estudio. El tomador de decisión puede gestionar el riesgo de uno o varios proyectos a partir de una perspectiva de la gestión de riesgos, como lo exponen Leggio, Bodde y Taylor (2006). También hay que reconocer que si bien la técnica de simulación Montecarlo es un método que se dicta en las aulas, por docentes expertos en evaluación de proyectos de inversión, las instituciones de educación superior aún no la utilizan como una herramienta para la evaluación de sus proyectos de inversión, ya que prefieren técnicas convencionales, como el análisis de costo/beneficio o análisis de sensibilidad.

Una vez que se tiene esta información se inicia el desarrollo del modelo de evaluación de proyectos de inversión bajo condiciones de riesgo, para que sea aplicado a una institución de educación superior.

El modelo de evaluación de proyecto de inversión formulado se estructura, en consecuencia, de la información preliminar; además, debe contar con la facilidad en su interpretación. Es importante resaltar que el factor diferenciador de esta investigación y del modelo formulado es la consideración de evaluación de un proyecto de inversión en condiciones de riesgo. 


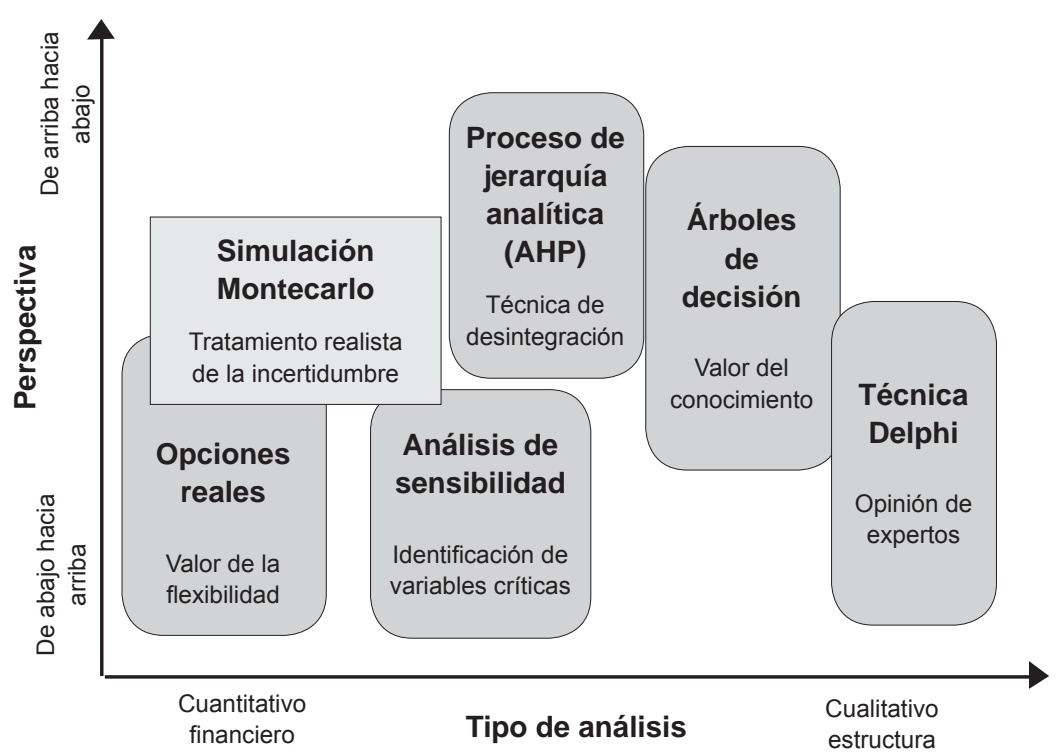

Figura 2. Perspectiva de análisis Adaptado de Leggio, Boddey Taylor (2006)

La modelación de parámetros estocásticos, como tradicionalmente se maneja en la evaluación de proyectos, se puede solucionar mediante el uso de distribuciones de probabilidad que se derivan de las evidencias registradas en el pasado y del conocimiento de las relaciones de las variables que la afectan. Sin embargo, aquellos factores, cuyo comportamiento es difícil predecir, se deben especificar con base en la experiencia y el juicio de quien toma la decisión (Bastidas y Ledezma, 2012). A partir de lo anterior se presenta, a continuación, la estructura del modelo para el desarrollo del modelo de evaluación de proyectos propuesto (figura 3), el cual muestra seis etapas, basadas en un ciclo de mejoramiento continuo, orientado a mejorar la competitividad del sector educativo, teniendo en cuenta que el factor diferenciador de esta investigación y del modelo formulado es la estimación de condiciones basadas en riesgo, cabe anotar que la última etapa, que es la toma de decisión, no se incluye en la explicación, pues a quien le corresponde la aplicación es a la institución; solamente se describe, para un mejor entendimiento (figura 3). De esta manera se mejora el proceso de decisión, considerando la posibilidad de continuar desarrollando la evaluación de proyectos de inversión bajo cualquier condición especial. 


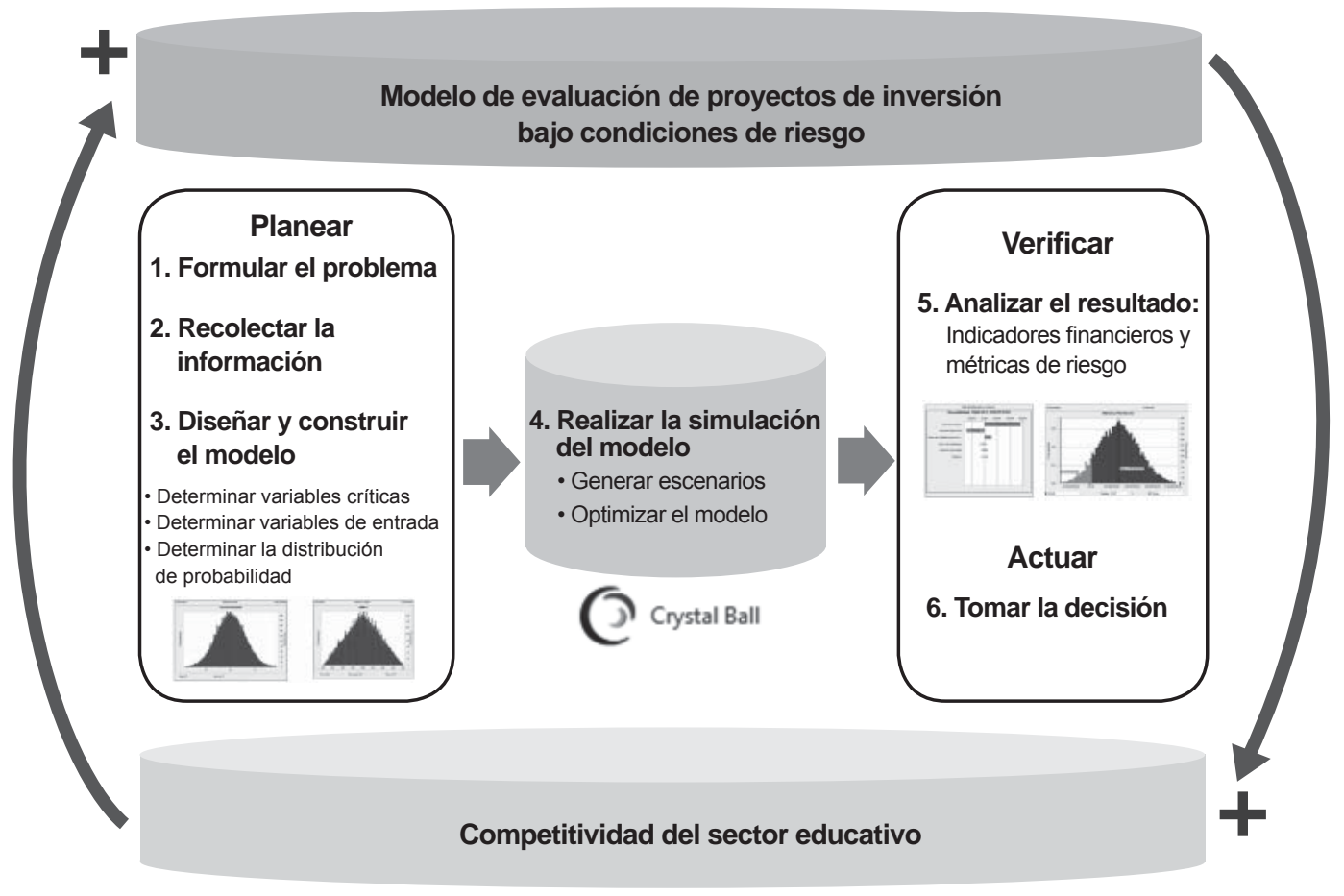

Figura 3. Modelo de evaluación de proyecto propuesto Elaboración propia

\section{- Etapa 1. Formulación del problema}

La formulación del problema se aplica en una institución de educación superior que pretende realizar la apertura de un nuevo programa académico, teniendo en cuenta las restricciones establecidas. La problemática está relacionada con la determinación de un modelo de evaluación de apertura de nuevos programas, considerando criterios financieros bajo condiciones de riesgo.

\section{- Etapa 2. Recolección de la información}

La información recolectada cobra relevancia debido a que se define si las variables de entrada se pueden aproximar a una distribución de probabilidad con un alto nivel de confianza. Los datos de entrada pueden ser determinísticos o estocásticos. Con los datos de entrada se pueden establecer las entradas y salidas de efectivo, en el modelo financiero. 


\section{- Etapa 3. Diseño y construcción del modelo}

El diseño y construcción del modelo determinístico propuesto se desarrolla en un ambiente que permita llevar a cabo las pruebas y ajustes necesarios, siendo flexible al momento de realizar la respectiva simulación. En esta actividad se debe considerar la distribución de probabilidad de cada uno de los parámetros claves para la evaluación del proyecto de inversión, la formulación de la función objetivo de riesgo, la rentabilidad y las restricciones establecidas.

a) Determinar variables críticas. En el modelo de decisión propuesto se ha definido como variables críticas los ingresos correspondientes al valor de las matrículas. El modelo de decisión ha considerado la construcción de un flujo de caja libre, el cual ha sido programado en Microsoft Office Excel. En dicho flujo se han considerado las variables asociadas a la medición de la apertura de un nuevo programa de pregrado en una institución de educación superior. Entre las variables establecidas se encuentran las siguientes: tasa de ingreso, tasa de deserción, valor de la matrícula e inscripción, gastos generales, valor de la hora cátedra y salarios, el salario mínimo mensual legal vigente, la inversión inicial y el nivel de deuda considerado.

El modelo del flujo de caja se ha elaborado con valores promedios de las variables críticas en primera instancia. A continuación se muestra el flujo de caja libre (figura 4), donde el costo de capital WACC se ha ajustado en un 6,8 \% (de acuerdo a datos proporcionados por el caso de estudio), con una participación de los socios correspondiente al $60 \%$ y deuda al $40 \%$, y un horizonte de cinco (5) años.

b) Determinar variables de entrada. Para la evaluación del modelo financiero se definieron las siguientes variables de entrada y sus respectivas funciones de distribución, realizadas con base en la información histórica de cada variable. Luego se observa la distribución de probabilidad para cada una de las variables de entrada definidas y formuladas en el modelo de evaluación del proyecto de inversión en una institución de educación superior (figura 5).

Se ha determinado, de acuerdo a la información considerada para la variable Salario Mínimo Mensual Legal Vigente (SMMLV), una variación porcentual que se ajusta a una distribución triangular con Mínimo 3,6 \%, Más Probable de 4 \% y Máximo de 4,4 \%. 


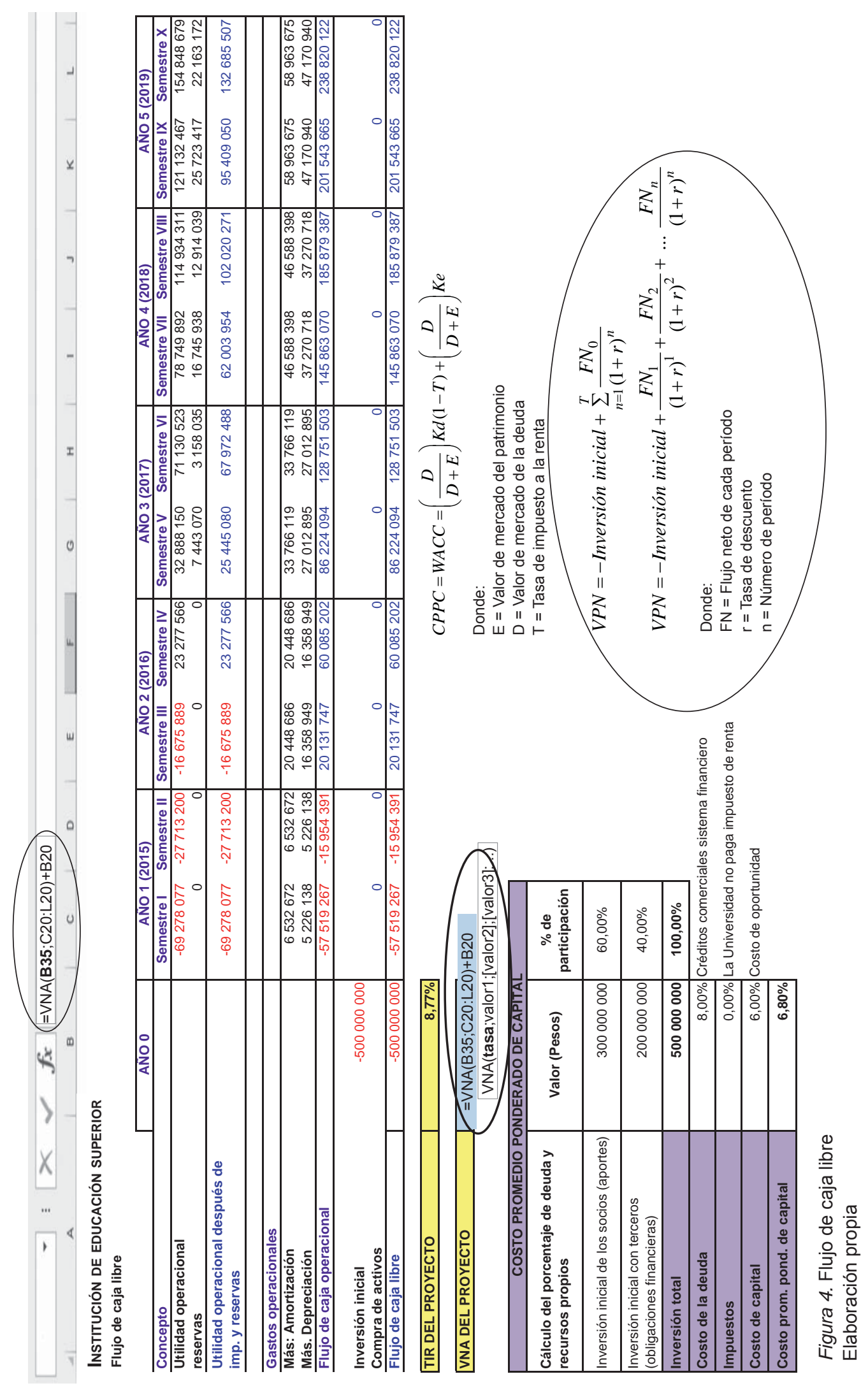




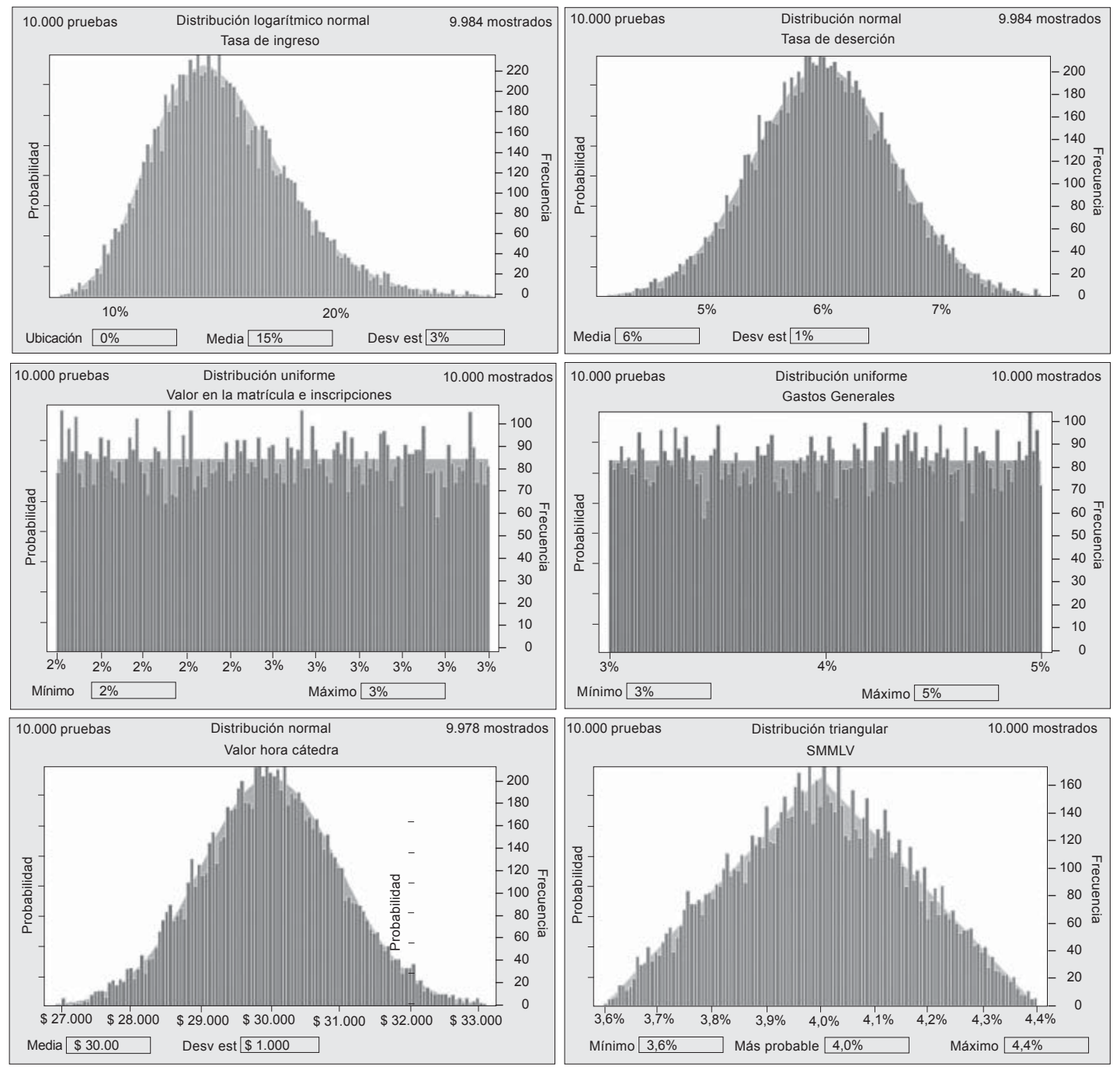

Figura 5. Distribución de las variables de entrada del modelo Elaboración propia

c) Determinar la distribución de probabilidad. Para determinar con certeza la distribución de probabilidad de los datos de entrada al modelo se utilizó las herramientas estadísticas definidas por Crystal Ball@, mediante la prueba de bondad y ajuste AndersonDarling, la cual es una prueba no paramétrica (Bravo y Sánchez, 2007). A continuación se presenta el resumen las distribuciones de probabilidad asignadas por Crystal Ball ${ }^{\circledR}$, teniendo en cuenta la información histórica (tabla 1).

La simulación de las variables y su aplicación dependen directamente de la forma en la que se construye el modelo y de las variables que se han tomado en cuenta con relación al sector educativo. 
Igualmente, se debe reconocer la posibilidad de que las relaciones entre las variables y el resultado no sean lineales. Por esta razón se ha realizado un análisis de sensibilidad de cada variable de manera independiente para poder identificar cuáles son las variables críticas, definiendo con esto en los datos de salida el valor presente neto (VPN) del proyecto calculado a partir de la función valor neto actual (VNA) del asistente de funciones del Excel.

\section{- Etapa 4. Simulación del modelo}

El modelo de decisión involucra una etapa de simulación Montecarlo, en el cual se realiza un análisis de las variables principales. La simulación Montecarlo se ha realizado a través del uso del software especializado Crystal Ball®, el cual permite la construcción del modelo en combinación con Excel.

Para esta aplicación se trata de demostrar cómo el cambio en una variable crítica afecta directamente el resultado del flujo de caja libre y por ende sus principales indicadores de desempeño financiero, para este caso en particular el VPN y la TIR. La simulación permite definir los diferentes escenarios de las variables más críticas de manera que sea posible determinar los rangos relativos en los cuales estas se pueden mover y en su efecto el indicador de desempeño financiero.

En este paso es necesario considerar algunas actividades para iniciar el proceso de simulación, como son: definir el número de simulaciones por realizar, verificar la relación entre el error estándar de la

Tabla 1

Resumen de las distribuciones de probabilidad de las variables de entrada

\begin{tabular}{lll}
\hline Variable & Tipo de distribución & Parámetros \\
\hline SMMLV & Distribución triangular & Min 3,6; Más probable 4; Max 4,4 \\
Tasa de deserción & Distribución normal & Media 6\%; Desvest 1 \\
Tasa de ingreso & Logarítmico normal & $\begin{array}{l}\text { Ubicación }=0,0 \% \text {; Media }=15,0 \% ; \\
\text { Desvest }=3,3 \%\end{array}$ \\
Gastos generales & Distribución uniforme & Mínimo 3; Máximo 5 \\
Valor hora cátedra & Distribución normal & Media 30.000; Desvest 1.000 \\
Valor de matrícula & Distribución uniforme & Mínimo 2; Máximo 3 \\
\hline
\end{tabular}

Elaboración propia 
media y la media obtenida para evaluar si el resultado es aceptable, definir la cantidad de escenarios debido a que la función estimada tenderá a representar a la función que caracteriza a toda la población, es decir, que a mayor número de experimentos más cercana será la función resultante del experimento (verdadera función), y por último, se debe correr la simulación y registrar los resultados.

Después de haber considerado estas actividades para iniciar el proceso de simulación, se muestra los datos obtenidos de la simulación con valores determinísticos (figura 6). Durante la preparación del

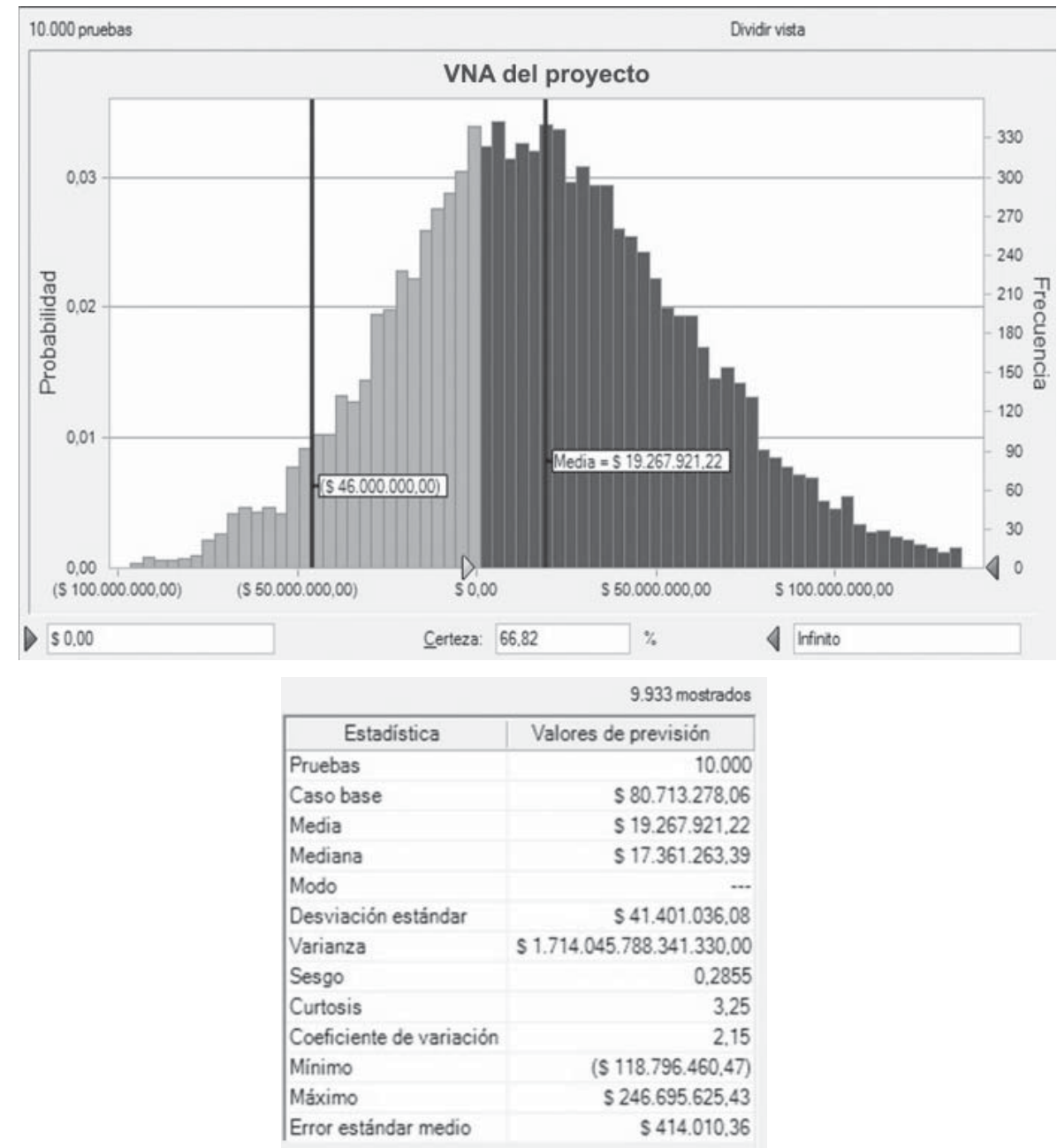

Figura 6. Resultado de la simulación del VPN con valores determinísticos Elaboración propia 


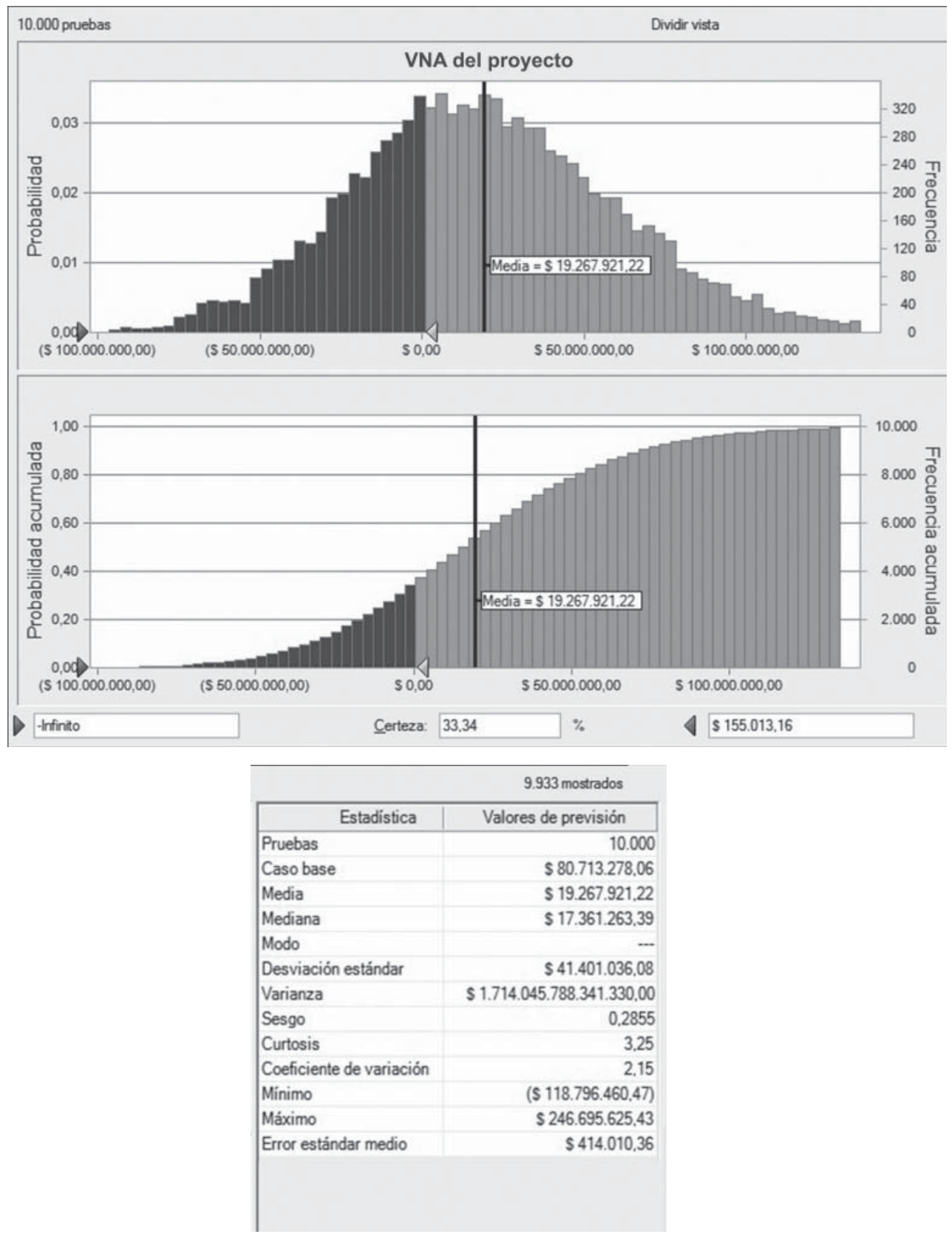

Figura 7. Valor presente neto en riesgo

Elaboración propia 
modelo de proyección de la variable resultado se ha tenido en cuenta las relaciones entre las diferentes variables causales (ingresos, costos, gastos, inversión, IPC, SMMLV, tasa de ingreso y tasa de deserción, etc.). Para este caso en particular, se ha considerado la investigación de un conjunto de datos que fueron modificados por procesos de confiabilidad de las cifras. Para algunas de estas se definió el tipo de distribución de probabilidad, de acuerdo a su comportamiento real, las cuales fueron estimadas, y a la experiencia de los tomadores de la decisión.

Igualmente, se observan los resultados obtenidos de la simulación con un número de 10.000 escenarios, justificado por la relación entre el Error Estándar Medio y la media del VPN, el cual arrojó un $2,1 \%,<5 \%$, lo cual se considera aceptable, debido a la ley de los grandes números, que establece que la función estimada tenderá a ser idéntica a la función que caracteriza a toda la población, es decir, que a mayor número de experimentos más cercana será la función resultante del experimento a la verdadera función (Manotas, 2009; Bastidas y Ledezma, 2012; Bravo y Sánchez, 2007). Cabe resaltar que para el desarrollo de la simulación se ha utilizado el software Crystal Ball ${ }^{\circledR}$ - Versión 11.1.2.3.500 (32 bits).

Mediante el análisis financiero tradicional se podría decir que el resultado es favorable, soportado en resultados de indicadores como el Coeficiente de Variación (215\%), indicando que el resultado involucra una gran variabilidad. Otros aspectos, poco considerados en las evaluaciones tradicionales, son los valores máximo y mínimo soportados por los análisis posteriores sobre el riesgo.

\section{- Etapa 5. Analizar resultados}

Después de realizada la simulación de las variables de entrada y salida se puede observar que el VPN del proyecto tiene un 33,34\% de probabilidad de ser menor que cero (figura 7). A su vez, también se puede analizar que existe un $66 \%$ de probabilidad de que la institución de educación superior tenga VPN mayor a cero, y esta probabilidad sugiere que la media del proyecto es $\$ 19,2$ millones sobre una base determinística de $\$ 80$ millones.

El Valor en Riesgo ( $\mathrm{VaR}$ ) es de $-\$ 46$ millones, con un nivel de confianza del 95,05\%, (figura 8), esto significa que la institución de educación superior tiene una probabilidad cercana al $5 \%$ de perder \$46 millones o más. 


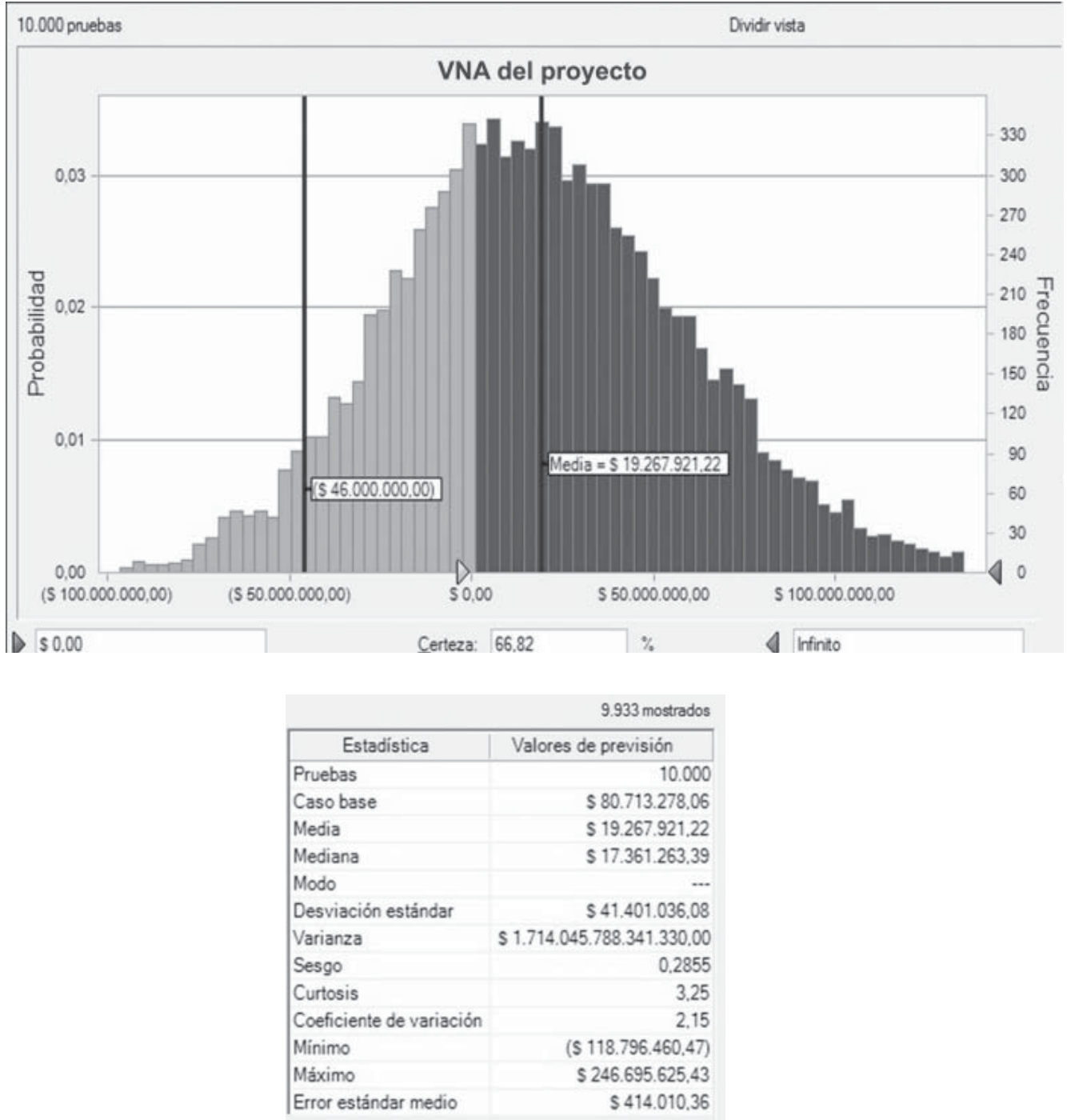

Figura 8. Probabilidad del valor presente neto en riesgo

Elaboración propia

De acuerdo con los resultados generados por el proyecto de inversión, un análisis posterior consiste en determinar las variables que toman mayor importancia en la evaluación del proyecto seleccionado, mediante la contribución de la varianza. Para este caso se puede observar que la variable Tasa de ingreso de los estudiantes tiene un impacto 


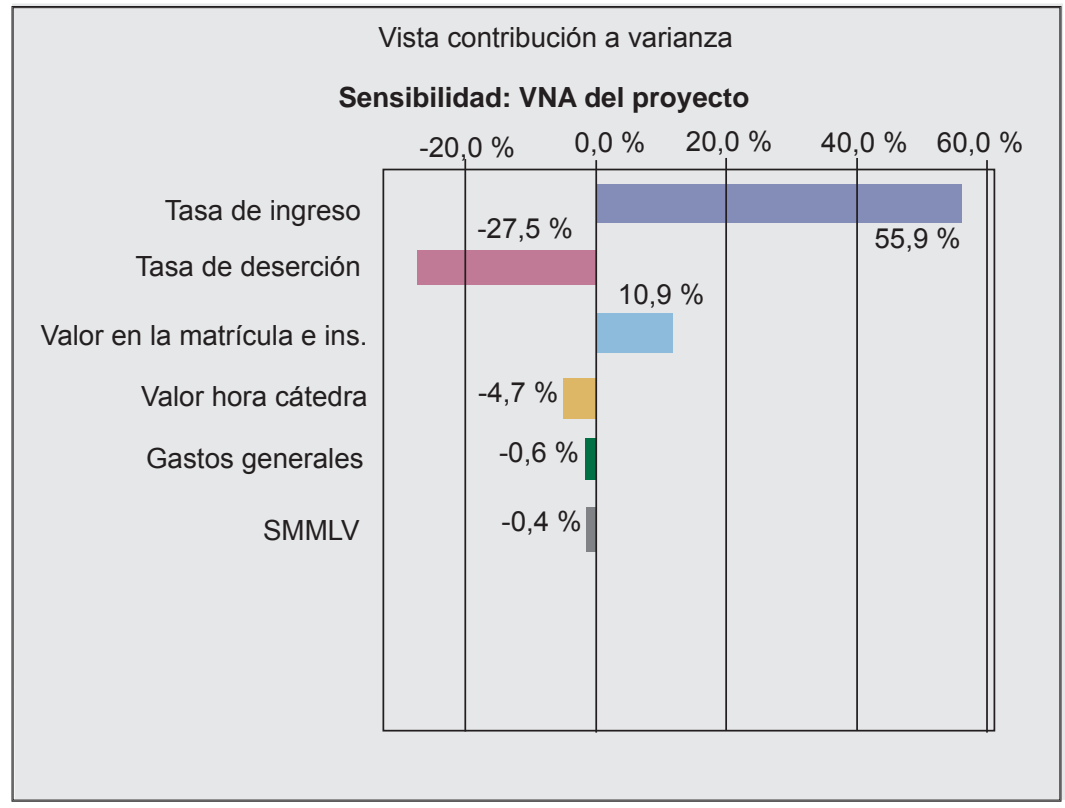

Figura 9. Análisis de sensibilidad de las variables del modelo Elaboración propia

positivo en la recuperación de la inversión, con un valor del 55,9 \%, (figura 9), lo contrario sucede con la variable Tasa de deserción, que es la que más contribuye de manera negativa al VPN, con un $27,5 \%$.

$\mathrm{Al}$ tener en cuenta los datos arrojados por la simulación se pueden definir las medidas de desempeño financiero, considerando su riesgo, para el caso particular del estudio se utilizará el VaR y CVaR, (figura 10). El Valor de Riesgo (VaR) propone una preparación ante el riesgo de $-\$ 46$ millones, pero el $\mathrm{CVaR}$, siendo más conservador, extiende como pérdida posible esperada -\$65 millones. Es importante anotar que el valor mínimo del ejercicio es de $-\$ 118$ millones, de allí que el valor esperado en este rango sea mayor al VaR.

a) Análisis de escenarios - Variable Tasa de ingreso

Si se modifica la tasa de ingreso y se pretende que este solo tenga una variación entre un mínimo y un máximo, dado que tiene una (1) variación estándar en los datos, se podría asumir que esta reducción en la variabilidad se incorpora al modelo y tiene un 


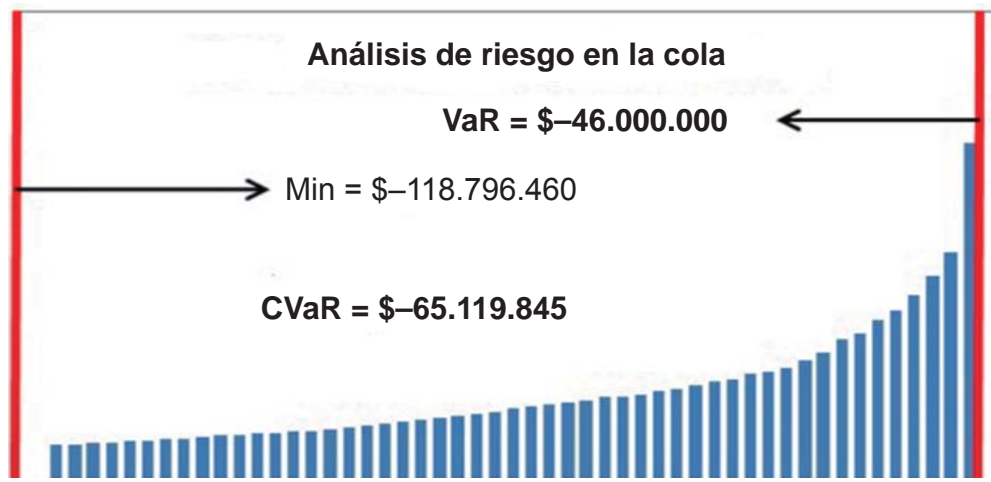

Figura 10. Comparativo entre el VAR y el CVAR Elaboración propia

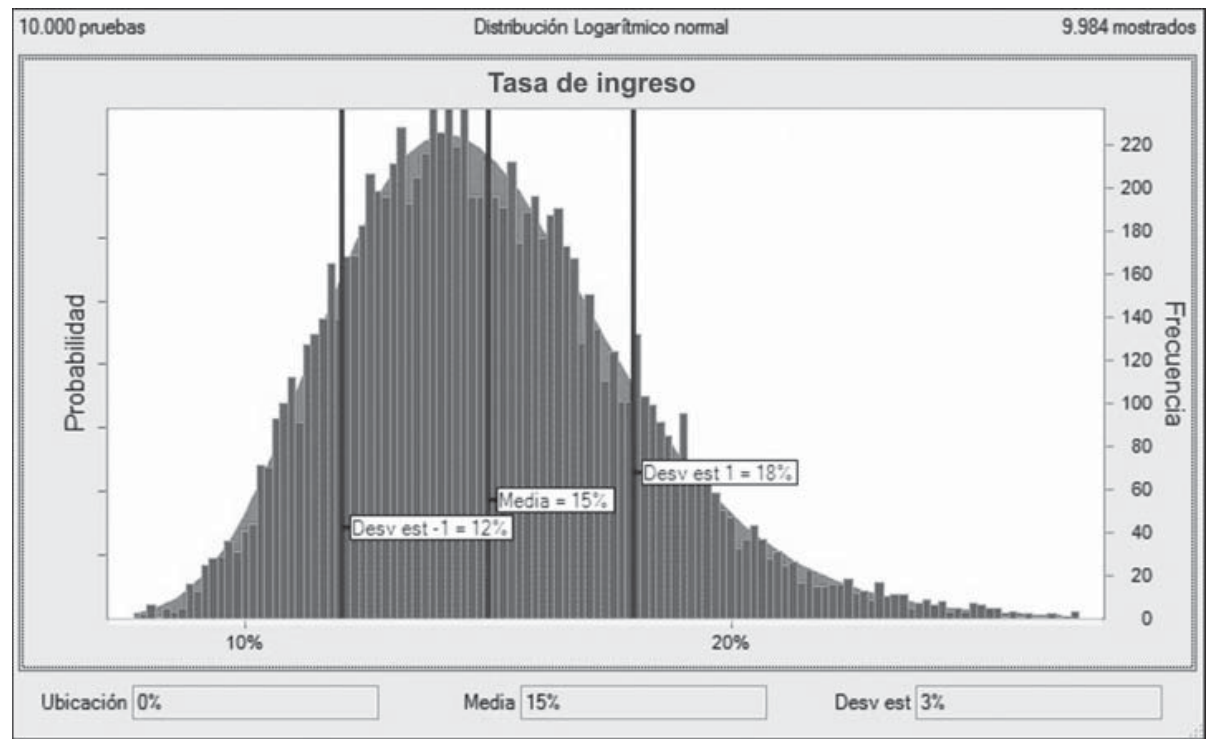

Figura 11. Parámetros estadísticos de la tasa de ingreso Elaboración propia 


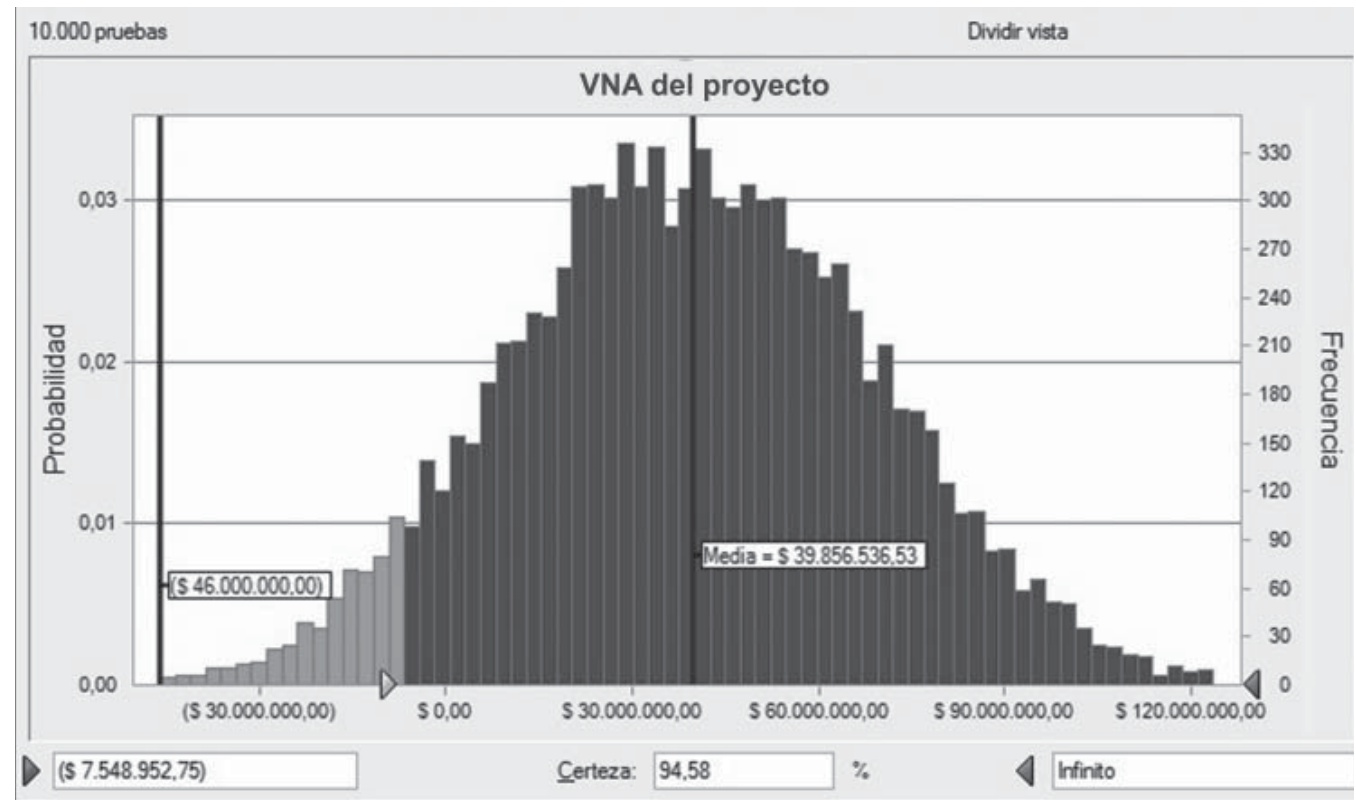

\begin{tabular}{|c|c|}
\hline & 9.946 mostrados \\
\hline Estadística & Valores de previsión \\
\hline Pruebas & 10.000 \\
\hline Caso base & $\$ 80.713 .278 .06$ \\
\hline Media & $\$ 39.856 .536 .53$ \\
\hline Mediana & \$ 39.447 .163 .87 \\
\hline Modo & - \\
\hline Desviación estándar & $\$ 29.850 .649 .87$ \\
\hline Varianza & \$ 891.061.297.684.224.00 \\
\hline Sesgo & 0.0865 \\
\hline Curtosis & 3.02 \\
\hline Coeficiente de variación & 0.7490 \\
\hline Minimo & (\$ 66,868.923,72) \\
\hline Máximo & S $170.238 .094,79$ \\
\hline Error estándar medio & $\$ 298.506 .50$ \\
\hline
\end{tabular}

Figura 12. Porcentaje del valor presente neto en riesgo Elaboración propia 
efecto sobre las mediciones de riesgo definidas. A continuación se presenta los datos originales de la variable "Tasa de ingreso de estudiantes" (figura 11), donde el resultado de modificar la variable redujo la variabilidad en el Coeficiente de Variación (CV) del orden de un $74 \%$ (figura 12).

\section{b) Análisis de escenarios - Variable Tasa de deserción}

Para el caso de la tasa de deserción se tuvo en cuenta el mismo supuesto para eliminar la variabilidad y solo era considerar los datos alrededor de una (1) desviación. En la figura 13 se presenta los datos originales de la variable "Tasa de deserción".

Manteniendo el mismo nivel de reducción de variabilidad utilizado en la corrida anterior se procedió a ajustar la variable, y se obtuvo los siguientes resultados antes y después del ajuste. Al correr nuevamente el proceso de simulación se puede ver que se ratifica la sensibilidad del modelo ante la variable "Tasa de deserción", pues los datos si bien fueron afectados en su variabilidad, el modelo mejora sus resultados en cuanto a su exposición a menor riesgo (figura 14).

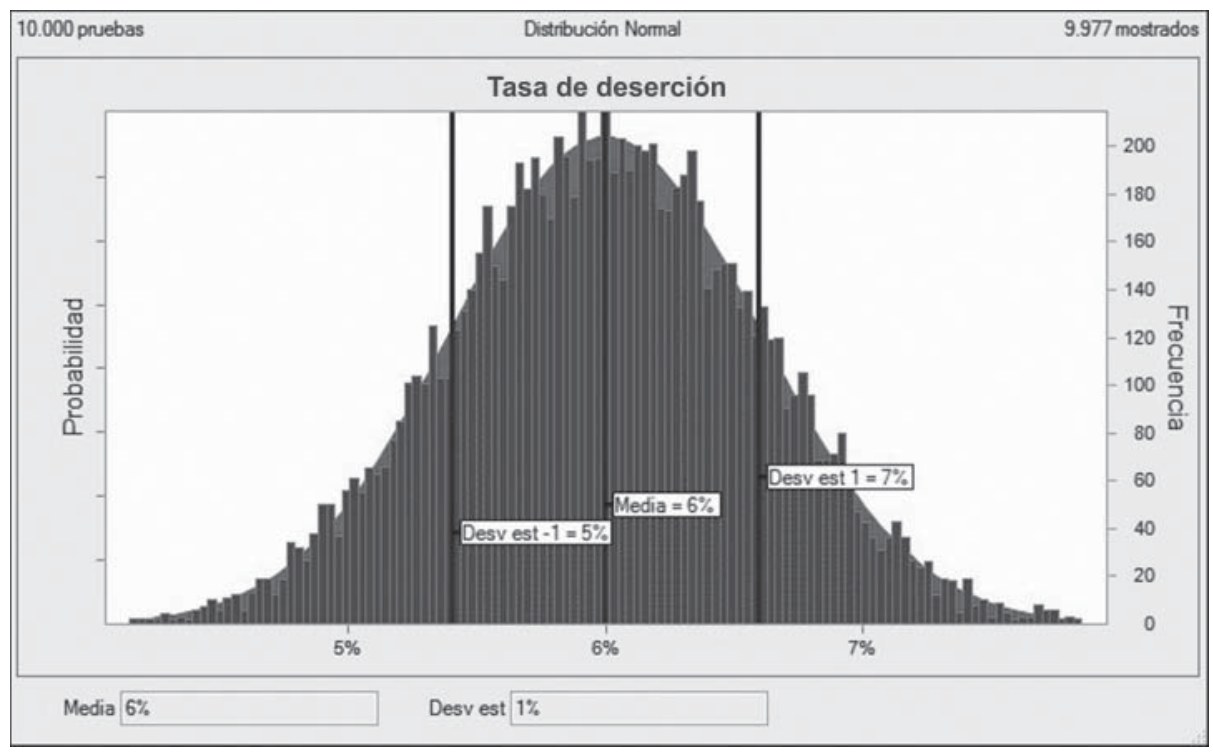

Figura 13. Parámetros estadísticos de la tasa de deserción Elaboración propia 


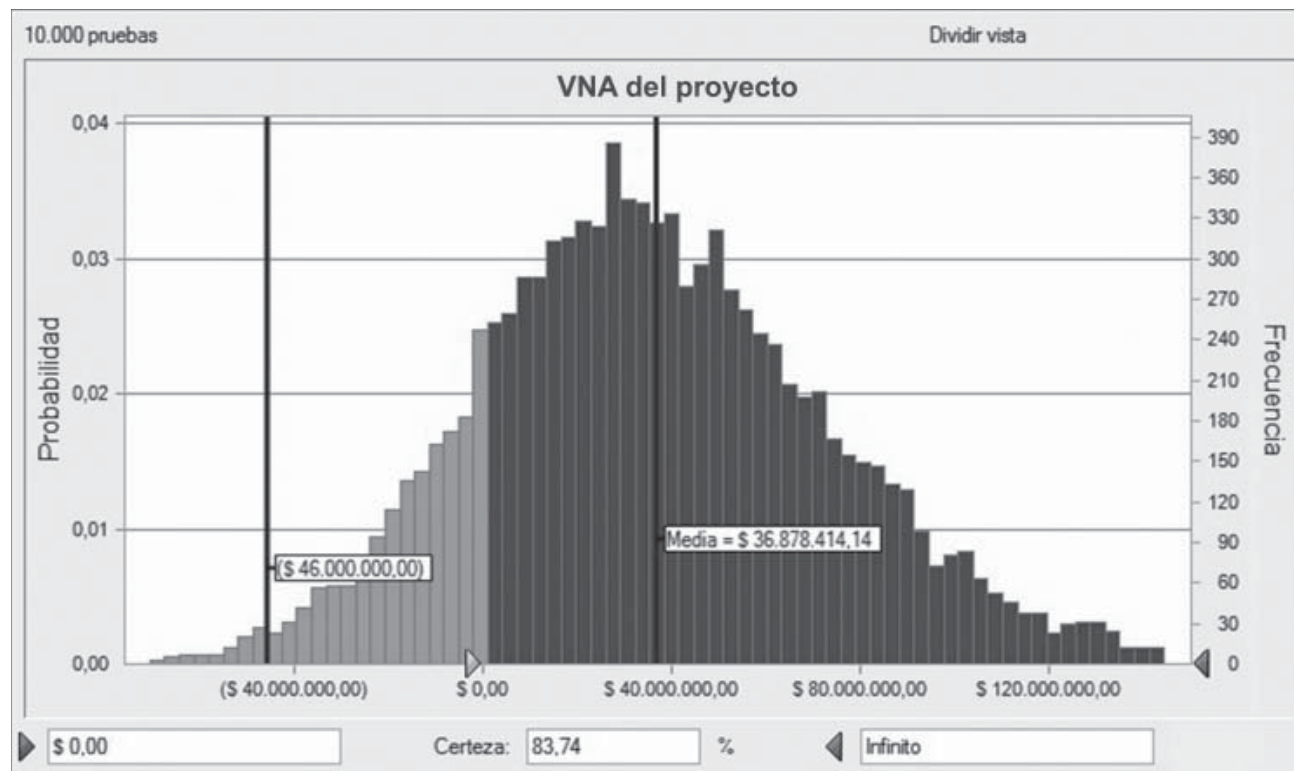

\begin{tabular}{|l|r|}
\hline \multicolumn{1}{|c|}{ Estadistica } & \multicolumn{1}{c}{9.923 mostrados } \\
\hline Pruebas & Valores de previsión \\
\hline Caso base & 10.000 \\
\hline Media & $\$ 80.713 .278 .06$ \\
Mediana & $\$ 36.878 .414 .14$ \\
\hline Modo & $\$ 34.368 .684 .97$ \\
\hline Desviación estándar & - \\
Varianza & $\$ 38.459 .702 .73$ \\
\hline Sesgo & $\$ 1.479 .148 .734 .442 .590 .00$ \\
\hline Curtosis & 0.3969 \\
Coeficiente de variación & 3.49 \\
\hline Minimo & 1.04 \\
Máximo & $\mathbf{( \$ 8 5 . 0 0 3 . 4 7 1 . 9 0 )}$ \\
Error estándar medio & $\$ 259.903 .113 .12$ \\
& $\$ 384.597 .03$ \\
\hline
\end{tabular}

Figura 14. Porcentaje del valor presente neto en riesgo

Elaboración propia 


\section{SIMULACIÓN DEL MODELO CON DIFERENTES ESCENARIOS}

Para generar un espacio comparativo de los distintos escenarios escogidos en la simulación del proceso, los resultados se detallan en la tabla 2.

Graficando los diferentes valores del VPN en cada uno de los percentiles, y de acuerdo con los escenarios propuestos, se obtiene que evaluando el proyecto de inversión analizado, el escenario más significativo es el escenario 2, el de menor incertidumbre; esto se reflejaen una menor exposición al riesgo, es decir, la línea del VPN es la que toma el menor valor negativo. Asimismo, podemos afirmar que el escenario 1, que corresponde a nuestra evaluación con toda la variabilidad, es el que mayor exposición a valores negativos presenta, como se observa en la figura 15.

Si se consideran los resultados obtenidos se puede determinar que la institución de educación superior debe generar controles y mecanismos adecuados de promoción y publicidad, de manera que disminuya la variabilidad presentada en la tasa de ingreso de los estudiantes, ya que con esto se impactará de manera positiva la volatilidad del VPN

Tabla 2

Cuadro comparativo de escenarios simulados

\begin{tabular}{lrrr}
\hline \multicolumn{1}{c}{ Métrica } & \multicolumn{1}{c}{ Escenario 1 } & \multicolumn{1}{c}{ Escenario 2} & \multicolumn{1}{c}{ Escenario 3} \\
\hline Caso Base & $\$ 80713278$ & $\$ 80713278$ & $\$ 80713278$ \\
VPN Medio & $\$ 19267921$ & $\$ 39856536$ & $\$ 36878414$ \\
Desvest & $\$ 41401036$ & $\$ 29850649$ & $\$ 38459702$ \\
CV & $215 \%$ & $74,9 \%$ & $104 \%$ \\
Min & $-\$ 1$ 187 964609 & $-\$ 66868923$ & $-\$ 850034711$ \\
Max & $\$ 246695625$ & $\$ 170238094$ & $\$ 259903113$ \\
VAR 95 \% & $-\$ 46000000$ & $-\$ 7548952$ & $-\$ 21933345$ \\
CVAR 95 \% & $-\$ 65119845$ & $-\$ 23703988$ & $-\$ 39516926$ \\
VPN O CV 95 \% & $33,34 \%$ & $9,00 \%$ & $16,26 \%$ \\
Var / Caso Base & $-57 \%$ & $-9,3 \%$ & $-27 \%$ \\
Cvar / Caso Base & $-80,50 \%$ & $-29,30 \%$ & $-48,90 \%$ \\
\hline
\end{tabular}

Elaboración propia 


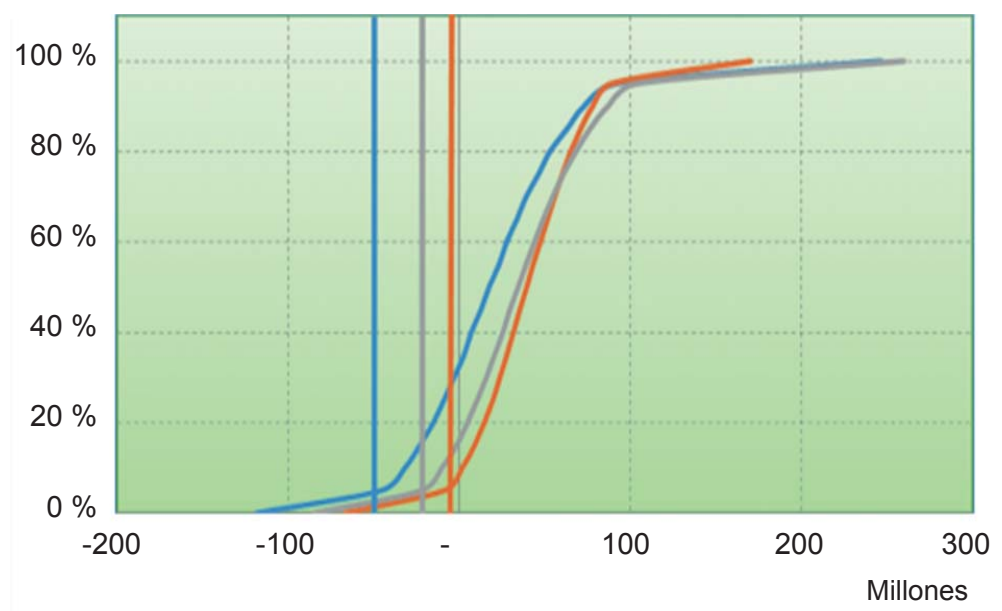

VNA escenario $1-$ VNA escenario $2 \longrightarrow$ VNA escenario 3

Figura 15. Distribución acumulada VPN de los escenarios simulados Elaboración propia

del proyecto y por ende se disminuirá el riesgo de la inversión, como se observa en la figura 15. También podría ser importante adaptar mecanismos de financiación que faciliten a los jóvenes de bajos recursos el acceso a los programas ofrecidos. No se puede perder de vista la tasa de deserción como un factor importante para incrementar el riesgo en el proyecto de inversión.

Adicionalmente, se puede utilizar la herramienta OptQuest de Crystal Ball ${ }^{\circ}$ para optimizar la composición accionaria del proyecto, de tal manera que se determine el porcentaje óptimo de la inversión de los socios y la deuda financiera, buscando menores niveles de riesgo en el desempeño financiero del proyecto. A continuación analizamos los siguientes tres escenarios en el proceso de optimización de la composición de la inversión:

\section{A. Minimizando el coeficiente de variación del VPN en riesgo}

$\mathrm{Al}$ optimizar la minimización del coeficiente de variación se revierte la composición de los componentes de la inversión, inicialmente determinados con la participación del $36 \%$ socios y $64 \%$ obligaciones financieras, como se puede observar en la figura 16, la cual corresponde al comportamiento del VPN de la figura 17. 


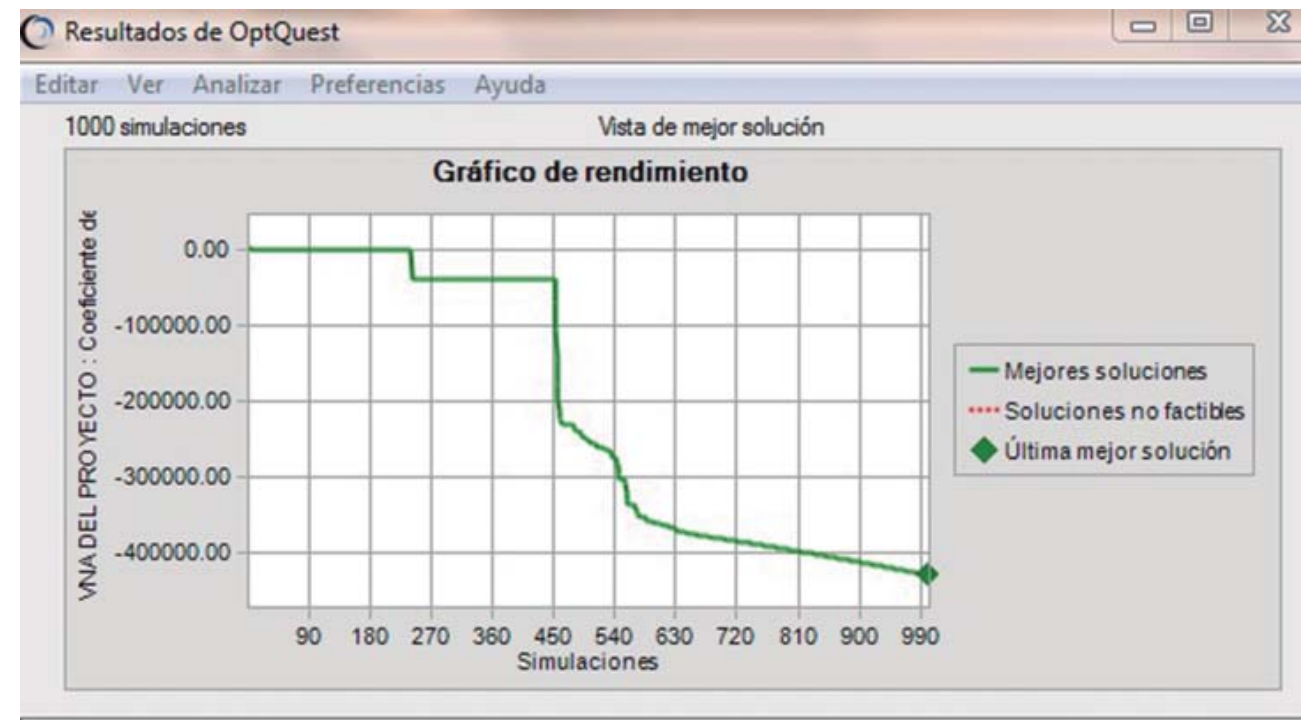

Mejor solución:

\begin{tabular}{|c|c|}
\hline \multicolumn{1}{|c|}{ Objetivos } & Valor \\
\hline Minimizar el/a Coeficiente de variación de VNA DEL PROYECTO & -430191.54 \\
\hline
\end{tabular}

\begin{tabular}{|c|c|}
\hline Requisitos & Valor \\
\hline
\end{tabular}

\begin{tabular}{|l|r|r|r|}
\hline \multicolumn{1}{|c|}{ Restricciones } & Lado izquierdo & & \multicolumn{1}{c|}{ Lado derecho } \\
\hline 'Fujo de Caja Libre'!D23 >=0 & $0,35533754645 \ldots$ & $>=$ & 0 \\
\hline 'Fujo de Caja Libre'!D24 >=0 & $0,64466350388 \ldots$ & $>=$ & 0 \\
\hline 'Fujo de Caja Libre'!D25 =100\% & $1,00000105033 \ldots$ & $=$ & 1 \\
\hline
\end{tabular}

\begin{tabular}{|l|r|}
\hline$-\quad$ Variables de decisión & Valor \\
\hline Inversión Inicial Con terceros (Obligaciones Financieras) & $64 \%$ \\
\hline Inversión Inicial de los Socios (Aportes) & $36 \%$ \\
\hline
\end{tabular}

Figura 16. Optimización bajo el coeficiente de variación

Elaboración propia 

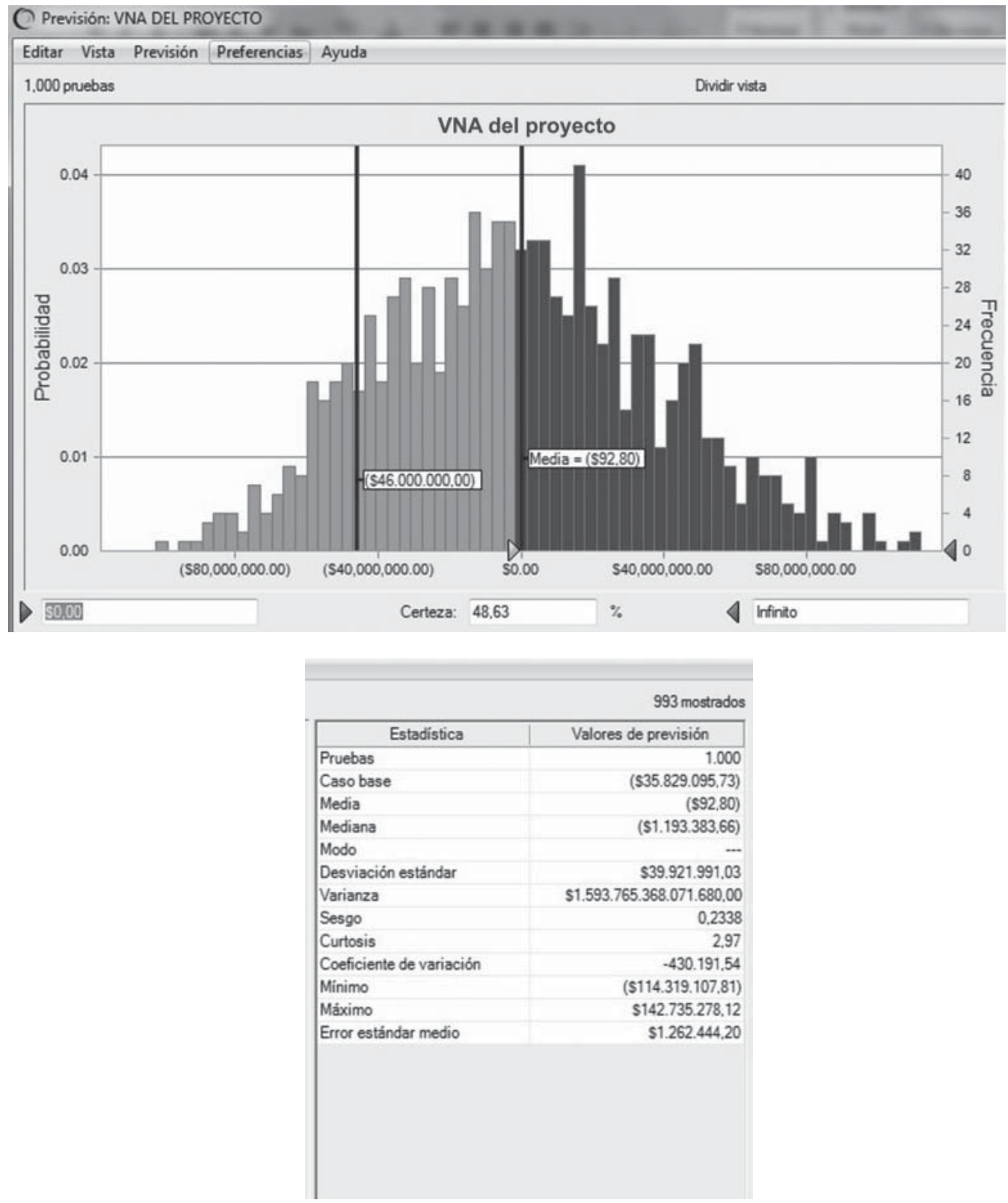

Figura 17. Comportamiento del VPN - escenario con coeficiente de variación Elaboración propia 


\section{B. Minimizando la desviación estándar del VPN en riesgo}

Optimizando la minimización de la desviación estándar la composición de los componentes de la inversión resulta 100 \% correspondientes a la obligación financiera, como se puede observar en la figura 18, la cual corresponde al comportamiento del VPN de la figura 19.

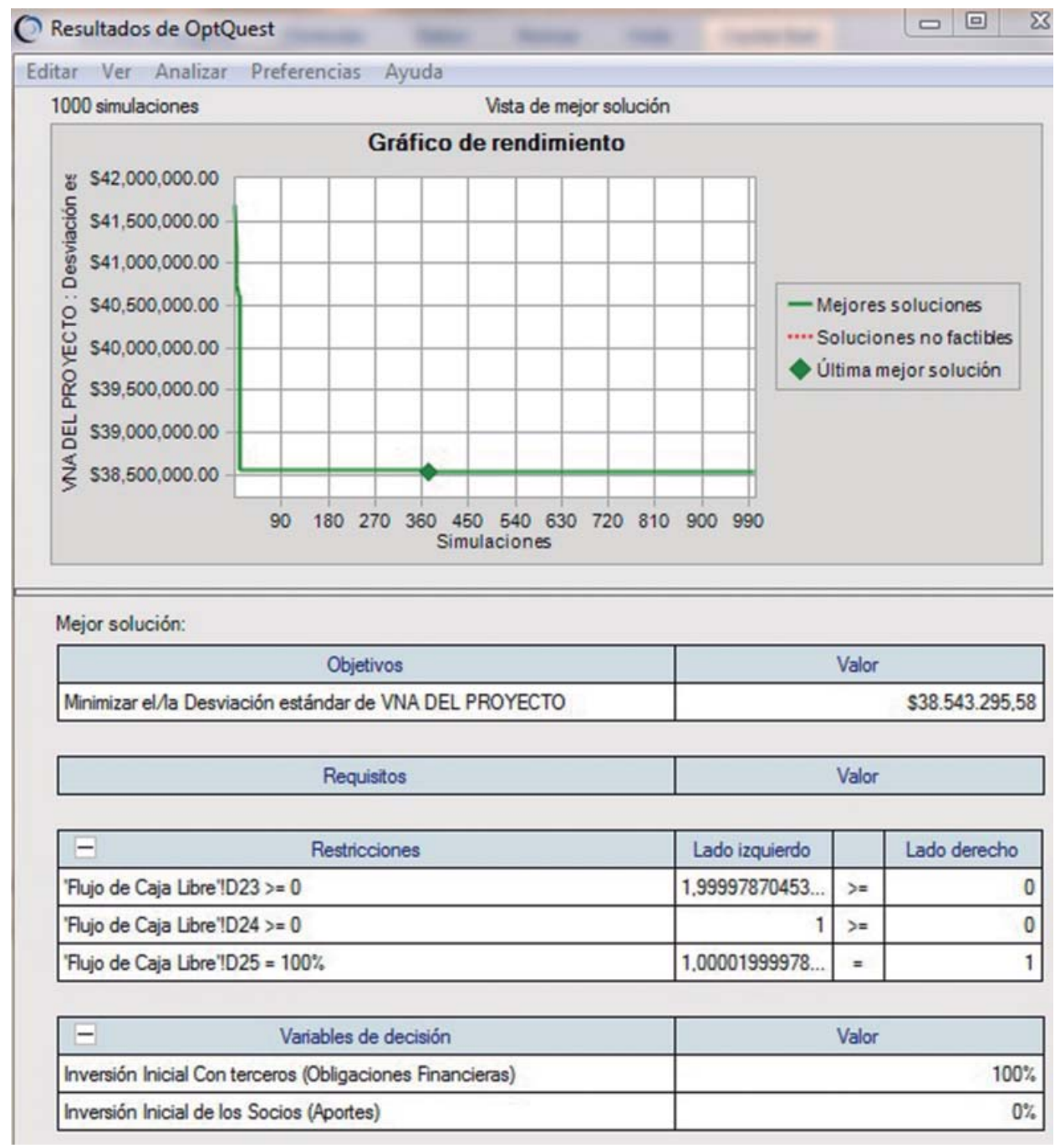

Figura 18. Optimización bajo la desviación estándar Elaboración propia 


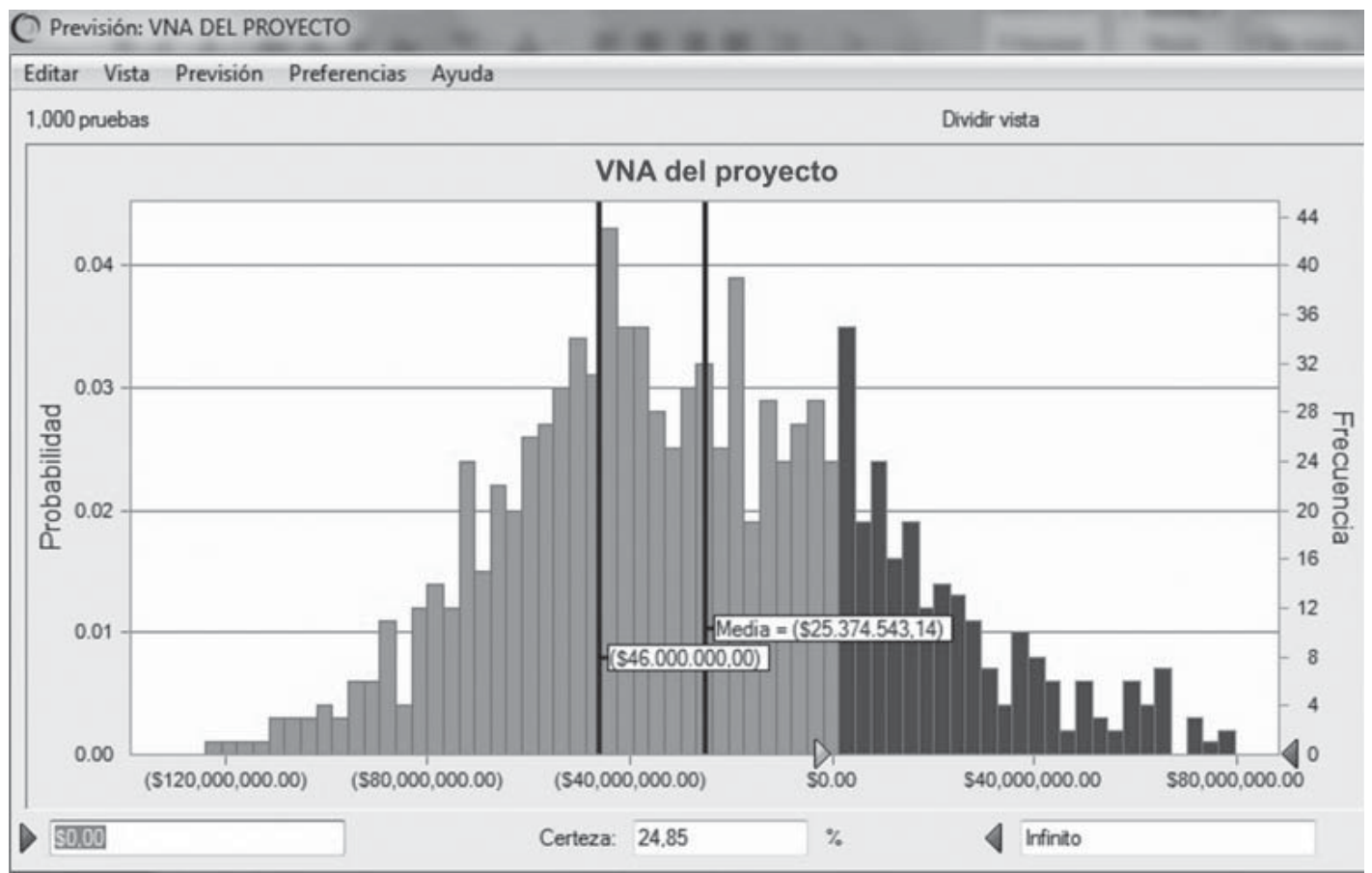

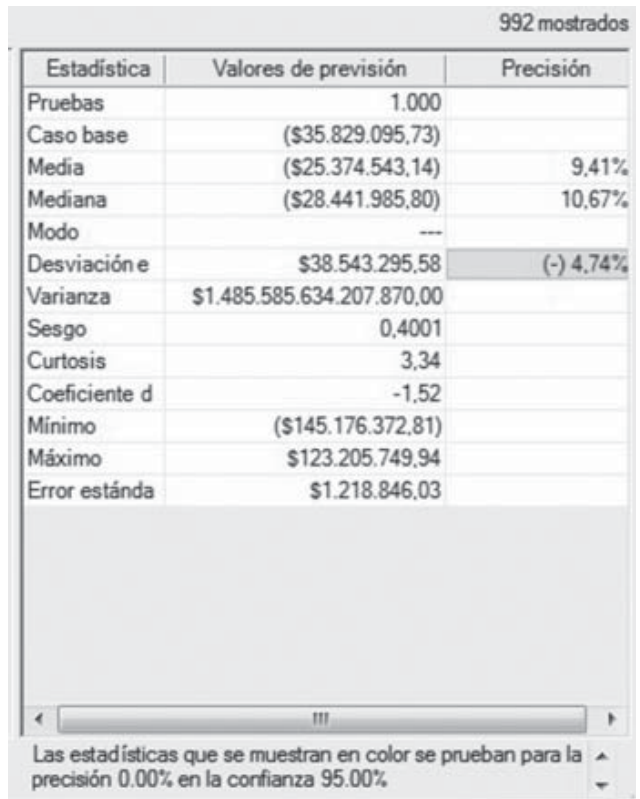

Figura 19. Comportamiento del VPN - escenario con coeficiente de variación Elaboración propia 


\section{Maximizando el valor esperado del VPN en riesgo}

Se puede concluir que la participación de los componentes de la inversión que mayor impacto positivo tiene sobre el VPN y que a la vez disminuye la exposición al riesgo es el escenario $0 \%$ obligaciones a terceros y $100 \%$ a socios, como se observa en la figura 20 , que se obtiene maximizando la media, según se aprecia en la figura 21.

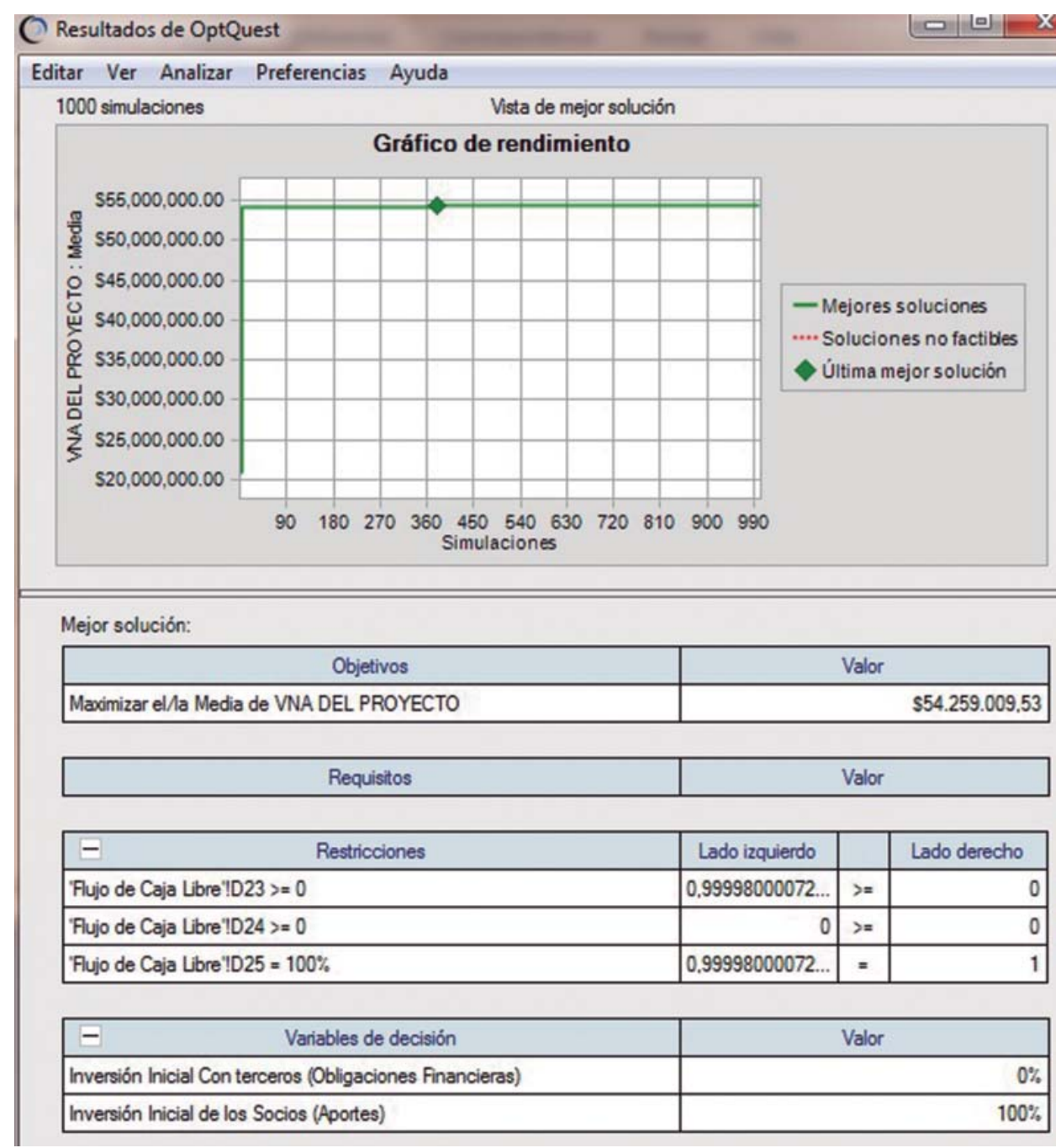

Figura 20. Optimización bajo la media Elaboración propia 

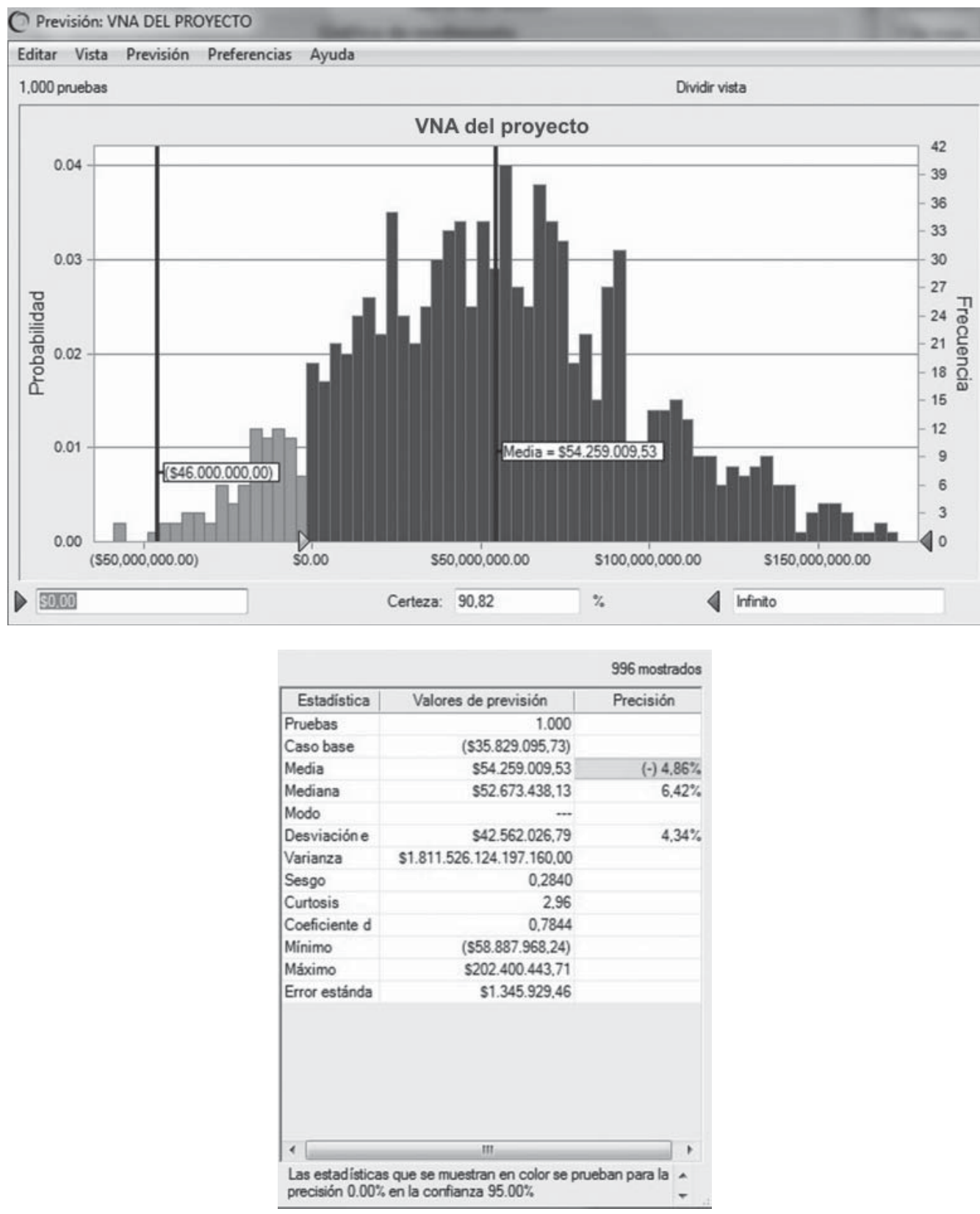

Figura 21. Comportamiento del VPN - escenario con la media Elaboración propia 
Con la simulación obtenida se puede afirmar que la aplicación de las herramientas que otorga la ingeniería financiera debe ser valorada para la evaluación de proyectos de inversión, más aún para el sector educativo, el cual promueve, a partir de la investigación, estos nuevos conocimientos.

Los resultados del análisis tradicional del VPN medio en los escenarios presentados corresponden a $\$ 19267$ 921, \$39 856536 y $\$ 36878$ 414, respectivamente, como se muestra en la tabla 2, así como el comportamiento de la desviación estándar y el coeficiente de variación del escenario 2 es el que presenta menores valores correspondientes al nivel de variabilidad. Por otro lado, el coeficiente de variación de los escenarios 1 y 3 está por encima de $100 \%$, lo cual demuestra la alta incertidumbre que tienen estos escenarios, independientemente de que los VPN sean positivos, los escenarios presentan altas probabilidades de tener VPN negativos, ya que el menor coeficiente de variación corresponde al escenario 2, con 74,9\%, reflejado también en valores mínimos y máximos.

Con relación a los valores mínimos y máximos de los escenarios 1 y 3 , presentados en la tabla 2, nos muestran que los datos están en un espectro más amplio, diferente a lo que sucede en el escenario 2 , en que los datos están más contraídos por un efecto en la reducción de la variabilidad cuando se eliminó la desviación estándar a los datos. Esto generó que la salida tenga una tendencia mucho más centralizada, evidenciándose en el coeficiente de variación.

Cuando se analizan las mediciones de riesgo, como el VaR, se evidencia que el escenario 2 es donde menor riesgo de pérdida tiene la institución de educación superior, diferente al escenario 1, que presenta el mayor riesgo de pérdida. Ahora bien, el CVaR, que es un indicador más conservador que el $\mathrm{VaR}$ al momento de valorar los riesgos asociados a la incertidumbre del proyecto, prepara el modelo con más riesgo de pérdida, manteniéndose el escenario 2 con menor riesgo de pérdida. Por otra parte, si revisamos el VPN en riesgo se puede observar que el escenario 2 presenta el $9 \%$ de probabilidad de tener valores menores a cero (0). Cabe anotar que CVaR es el valor esperado entre el mínimo y el VaR.

\section{CONCLUSIONES}

Es importante considerar que en el análisis tradicional de proyectos el tomador de la decisión se ve enfrentado a tomar decisiones con varia- 
bles que presentan comportamientos determinados, lo cual claramente es una visión corta de lo que podría ser el resultado final del proyecto. En caso contrario, si el tomador de decisión tuviera en cuenta la variabilidad y la incertidumbre asociada al comportamiento estocástico de las variables se puede determinar su grado de impacto sobre la métrica financiera, en este caso el VPN.

- Se demuestra que la variabilidad y la incertidumbre tienen una incidencia directa sobre los niveles de riesgo, a su vez que permite la determinación de las variables que más impactan de forma positiva o negativa, pues en la metodología propuesta aparecen indicadores: el coeficiente de variación, el VaR, el CVaR, el mínimo y el máximo como métricas capaces de brindar información adicional al tomador de decisión, como lo es el nivel de riesgo al que se debe preparar o la posibilidad de tener éxito o fracaso.

- La simulación Montecarlo aparece como una herramienta poderosa, capaz de producir una gran cantidad de escenarios, donde cada variable toma valores dentro de su distribución de probabilidad y las salidas por la ley de los grandes números toma una distribución normal, con todas sus bondades en el análisis, como es el uso de la probabilidad.

- La formulación del modelo relaciona las mediciones actuales de desempeño en la evaluación de proyectos de inversión de una institución de educación superior, con métricas financieras enfocadas en la generación de valor, incorporando mediciones de riesgo e incertidumbre, como el VaR y el CVaR.

\section{REFERENCIAS}

Bastidas, E., y Ledezma J. (2012). Modelo de evaluación del desempeño financiero en cadenas de suministros en condiciones de incertidumbre. Tesis de grado. Colombia: Universidad del Valle.

Bock, K., y Trück S. (2011). Assessing uncertainty and risk in public sector investment projects. Technology and Investment, 2, 105-23.

Bravo, O., y Sánchez, M. (2007). Gestión integral de riesgos. (2. ${ }^{a}$ ed.). Vol.1. Bogotá: Consorcio Gráfico.

Cremin, P., y Nakabugo, M. (2012). Education, development and poverty reduction: a literature critique. International Journal of Educational Development. Science Direct, 32, 499-506. 
Gölpek, F. (2012). Price of higher education and individual demand. Procedia - Social and Behavioral Sciences, 41, 349-356.

Jara, R., y Melgar, J. (2007). VaR vs. CVaR. ¿Qué estimador se ajusta mejor al riesgo de mercado de renta variable en el Perú? Working Paper. Recuperado de http://www.griskm.com/articulo02.pdf

Leggio, K., Bodde, D., y Taylor, M. (2006). Chapter VI. Real options and Monte Carlo Simulation versus Traditional DCF Valuation in Layman's Terms. En Managing Enterprise Risk - What the electric industry experience implies for contemporary business. Elsevier Global Energy Policy and Economics Series. Great Britain.

Manotas, D. (2009). Optimal economic project selection under uncertainty: an illustration from an utility company. Ingeniería y Competitividad. Vol. 11, 41-52.

Manotas, D., y Toro, H. (2008). Evaluación de proyectos utilizando el criterio valor presente neto en riesgo (VPN en riesgo). Revista Facultad de Ingeniería Universidad de Antioquia, 49, 199-213.

Melo, L. F., y Becerra, O. (2004). Medidas de riesgo, características y técnicas de medición: una aplicación del VaR y el ES a la tasa interbancaria de Colombia. Bogotá: Banco de la República, Gerencia Técnica.

Ortiz, H., y Ortiz, D. A. (2009). Flujo de caja y proyecciones financieras con análisis de riesgo. (2. ${ }^{\mathrm{a}}$ ed.). Bogotá: Universidad Externado de Colombia.

Schwab, K., y Sala-i-Martin, X. (2013). The Global Competitiveness Report 2013-2014. World Economic Forum. Recuperado de http://www3. weforum.org/docs/WEF_GlobalCompetitivenessReport_2013-14.pdf

Vélez, I. (2003). Decisiones empresariales bajo riesgo e incertidumbre. Vol. 1. Bogotá: Grupo Editorial Norma.

Wenyi, L. (2008). Uncertainty study of financial evaluation of project investment. IEEE, 2, 285-289. 